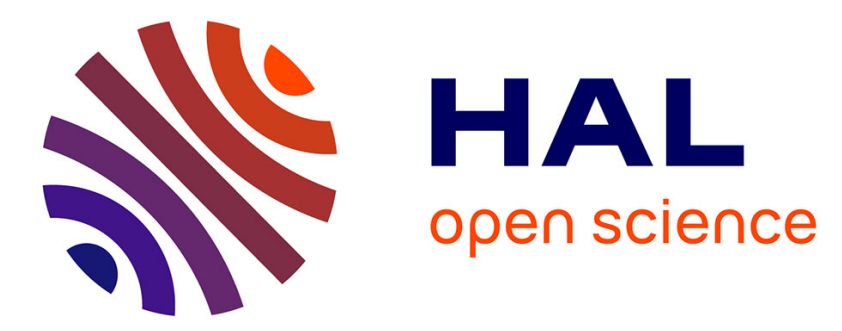

\title{
Extension of Biot's theory of wave propagation to frozen porous media
}

Philippe Leclaire, Frédéric Cohen-Ténoudji, Jaime Aguirre-Puente

\section{To cite this version:}

Philippe Leclaire, Frédéric Cohen-Ténoudji, Jaime Aguirre-Puente. Extension of Biot's theory of wave propagation to frozen porous media. Journal of the Acoustical Society of America, 1994, 10.1121/1.411336 . hal-01324844

\section{HAL Id: hal-01324844 \\ https://hal.science/hal-01324844}

Submitted on 1 Jun 2016

HAL is a multi-disciplinary open access archive for the deposit and dissemination of scientific research documents, whether they are published or not. The documents may come from teaching and research institutions in France or abroad, or from public or private research centers.
L'archive ouverte pluridisciplinaire HAL, est destinée au dépôt et à la diffusion de documents scientifiques de niveau recherche, publiés ou non, émanant des établissements d'enseignement et de recherche français ou étrangers, des laboratoires publics ou privés. 


\title{
Extension of Biot's theory of wave propagation to frozen porous media
}

\author{
Ph. Leclaire and F. Cohen-Ténoudji \\ L.U.A.P. Université D. Diderot (Paris 7), 2 place Jussieu, 75251 Paris Cedex 05, France \\ J. Aguirre-Puente \\ Centre de Géomorphologie du C.N.R.S., 24 avenue des Tilleuls, 14000 Caen, France
}

\begin{abstract}
An extension of Biot's theory is proposed for frozen porous media where the solid substrate, ice particles, and unfrozen water can coexist. Elastic, kinetic, and dissipation energy densities are written using the results of continuum mechanics, then the equations of propagation are deduced with the help of Lagrange's equations and Hamilton's least-action principle. The ice parameters are introduced in the model in addition to those used in Biot's theory. It appears that only the percolation theory is able to describe the transition of the ice matrix between the continuous state and the discontinuous state during a freezing or a thawing process. The resolution of the equations of propagation lead to the existence of three longitudinal and two transverse modes. Their velocities and attenuations are calculated as functions of the physical parameters of the medium. Independently, a thermodynamical argument is developed which allows the mechanical properties to be related to temperature. Experimental results are briefly presented to confirm the theoretical predictions.
\end{abstract}

\section{INTRODUCTION}

Water-saturated porous media encountered in geophysics, civil engineering, or the food industry are sometimes submitted to low temperatures during freezing processes. The thermodynamical study of these media has shown that ice and unfrozen water can coexist far below $0{ }^{\circ} \mathrm{C}$ in proportions depending on granulometry and temperature. More precisely, it is known that for a given negative celsius temperature a finely dispersed frozen medium will contain more unfrozen water than a coarse one. As a consequence, the mechanical properties of frozen porous media are strongly dependent on granulometry and temperature.

Various methods exist to study the freezing of porous media. Electrical conductivity, nuclear magnetic resonance, or calorimetry have been successfully used to bring out the unfrozen water content (see Deschatres et al., 1989). Acoustic wave propagation which is sensitive to mechanical variations provides a nondestructive method for the characterization of frozen porous media. It consists in propagating elastic waves in the medium in order to deduce information from the observation of the received signals. During a freezing or a thawing process the wave velocities can be measured as a function of temperature (Timur, 1968). The experimental results may be associated with a theoretical model to characterize the mechanical properties and their relationships with the thermodynamical state of the medium.

Unfortunately, the semiempirical models developed until now are not elaborate enough to describe all the experimental results. A model of weighted average of bulk moduli has been developed and seems to be useful for unconsulidated media; another one which consists in averaging the waves slownesses would apply for consolidated media (Deschatres et al., 1989). But still no complete theory exists that would explain all the experimental results in a unified way. Biot $(1956 a, b)$ has established a complete theory of the propagation of elastic waves in fluid-saturated porous solids which has been tested and confirmed (Plona and Johnson, 1980; Berryman, 1980; Bacri and Salin, 1982) and successfully applied for the interpretation of seismic waves observed in geophysics (Bourbié et al., 1987). Nevertheless, this theory is not usable for the freezing of porous media without adaptation. In this paper, an extension of Biot's theory is proposed to include the phase transition of the interstitial fluid between liquid state and solid state. For this, one may consider the existence of two solids (solid substrate and ice matrix) and a liquid (unfrozen water) saturating the two overlapped skeletons.

Several assumptions are made but two have a major importance for the model. The first one concerning the porous medium thermodynamics is that there is no direct contact between solid substrate and ice. The second essential assumption concerns the propagation: the wavelengths are supposed to be greater than the minimum length of homogenization in order that the materials properties are averaged and are those of effective continuous media. Hence, using the formalism of continuum mechanics, potential, kinetic, and dissipation energy densities per unit volume of porous material are expressed with emphasis on elastic, inertial, and viscous coupling between the effective phases. The equations of propagation deduced from Hamilton's least-action principle provide three longitudinal and two transverse solutions.

Biot's generalized elastic coefficients $\left(R_{i j}\right)$ and mass densities $\left(\rho_{i j}\right)$ defined in the energy terms are expressed with the help of Biot's results (Biot and Willis, 1957; Biot, 1962). The viscosity effect on the transmission of shear strengths in liquids is included. Furthermore, different theories of hetero- 
geneous material elasticity are tested to evaluate the elastic coefficients of the two solid skeletons (substrate and ice). It appears that only a percolation model is able to describe the variations of the ice matrix rigidity during a freezing or a thawing process.

A numerical solution of the equations of propagation allows one to plot the velocity and attenuation coefficient of three compressional and two shear waves as functions of unfrozen water content and frequency. Whereas Biot's theory brings out the existence of two regimes separated by a characteristic frequency depending on the capillary pore radius, in this problem the characteristic frequency is a function of unfrozen water thickness. With the help of a thermodynamical relationship, it is possible to plot the shape of the velocity curves as a function of temperature. Experimental results are briefly presented in order to make a first comparison with the theoretical predictions.

\section{THEORY}

The results presented here are parts of doctoral works of one of us (Leclaire, 1992). A summary of the model and applications to frozen soils have been presented (Leclaire et al., 1991, 1993).

\section{A. Model hypothesis}

As in Biot's theory the medium is supposed to be statistically isotropic, totally saturated, and with a uniform and connected porosity. The porosity $\varepsilon$ is given by the ratio of the volume occupied by the pores to the total volume. We may consider a water saturated porous medium at temperatures below $0^{\circ} \mathrm{C}$. It is known that unfrozen water and ice can coexist at negative celsius temperatures in finely dispersed frozen media (see Aguirre-Puente and Bernard, 1978). Hence three phases may be considered: a solid substrate, an ice matrix, and unfrozen water saturating the two overlapped matrices. The volumic proportions of solid $\phi_{s}$, of unfrozen water $\phi_{w}$, and of ice $\phi_{i}$ are given, respectively, by the ratio of the volume of each phase to the total volume. The mechanical properties of interstitial water are considered to be those of free water. This supposes that the proportion of adsorbed water on solid substrate (see Defay and Prigogine, 1957) is small compared to the total proportion of unfrozen water. It is also assumed that the total water proportion (unfrozen water and ice) is constantly equal to the porosity

$$
\phi_{w}+\phi_{i}=\varepsilon \text {. }
$$

Thus an elementary relationship always exists between the constituent proportions

$$
\phi_{s}+\phi_{w}+\phi_{i}=1 \text {. }
$$

An important assumption is that there is no direct contact between solid substrate and ice except in the limit case of a totally frozen medium. The problem then becomes much simpler and its mathematical formulation is possible. Indeed, it is known that the adsorption mechanism in finely dispersed frozen media (Aguirre-Puente and Bernard, 1978) implies the existence of a layer of unfrozen water around the solid particles isolating them from ice (Fig. 1).

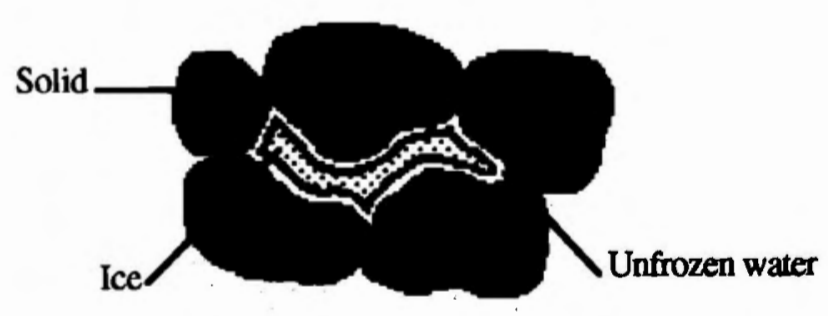

FIG. 1. Ice formation in frozen porous media.

Concerning the propagation of elastic waves, the wavelengths are supposed to be greater than the minimum length of homogenization for which the physical properties of the medium are averaged and are those of a continuous medium (see Leclaire, 1992). As a consequence one may study the propagation in continuous effective phases equivalent to the real inhomogeneous phases. It is then possible to define the physical parameters classically used in continuum mechanics: the potential, kinetic, and dissipation energy densities and also the stresses, the strains, and the momenta. All these parameters may be defined for each effective phase per unit volume of porous material. It may be noticed that the concept of effective phase has a significance at macroscopic scale only. In the following presentation, the effective solid, the unfrozen water as an effective fluid and the ice as a second effective solid will sometimes be, respectively, designated by the terms of solid, water, and ice. The indices 1,2 , and 3 will be, respectively, used referring to them in the formulas.

\section{B. Potential energy density V}

When an elastic wave propagates, the porous medium is submitted to local deformations and temperature variations; the energy function used to describe these phenomena should be the free energy. However for small temperature variations, the potential energy $V\left(\varepsilon_{i j}^{(1)}, \varepsilon_{i j}^{(2)}, \varepsilon_{i j}^{(3)}\right)$ can be considered as elastic energy and depends only on the strain tensors of solid $\varepsilon_{i j}^{(1)}$, of water $\varepsilon_{i j}^{(2)}$, and of ice $\varepsilon_{i j}^{(3)}$. The strain tensors are written as functions of the displacement vectors $u_{1}, u_{2}$, and $u_{3}$. For solid and ice:

$$
\varepsilon_{i j}^{(1)}=\frac{1}{2}\left(\operatorname{grad} u_{1}+\operatorname{grad}^{T} u_{1}\right)
$$

and

$$
\varepsilon_{i j}^{(3)}=\frac{1}{2}\left(\operatorname{grad} u_{3}+\operatorname{grad}^{T} u_{3}\right),
$$

where grad is the space derivative operator with respect to the three space coordinates and $\operatorname{grad}^{T}$ the transposed operator. Water being statistically isotropic and without shear deformations its strain tensor is diagonal and can be expressed with the first invariant $\theta_{2}=\varepsilon_{k k}^{(2)}$ (Einstein's convention of summation on repeated indices is used) called the dilatation:

$$
E_{i j}^{(2)}=\left(\begin{array}{ccc}
\theta_{2} / 3 & 0 & 0 \\
0 & \theta_{2} / 3 & 0 \\
0 & 0 & \theta_{2} / 3
\end{array}\right) .
$$

For small deformations from the equilibrium state generally associated with elastic waves and in absence of exter- 
nal forces, the problem is linearized developing $\mathbf{V}$ to the second order:

$$
\begin{aligned}
\mathbf{V}\left(\varepsilon_{i j}^{(1)}, \theta_{2}, \varepsilon_{i j}^{(3)}\right)= & \mathbf{V}(0,0,0)+\frac{1}{2} \frac{\partial^{2} \mathbf{V}}{\partial \varepsilon_{i j}^{(1)} \partial \varepsilon_{k l}^{(1)}} \varepsilon_{i j}^{(1)} \varepsilon_{k l}^{(1)} \\
& +\frac{1}{2} \frac{\partial^{2} \mathbf{V}}{\partial \theta_{2}^{2}} \theta_{2}^{2}+\frac{1}{2} \frac{\partial^{2} \mathbf{V}}{\partial \varepsilon_{i j}^{(3)} \partial \varepsilon_{k l}^{(3)}} \varepsilon_{i j}^{(3)} \varepsilon_{k l}^{(3)} \\
& +\frac{\partial^{2} \mathbf{V}}{\partial \varepsilon_{i j}^{(1)} \partial \theta_{2}} \varepsilon_{i j}^{(1)} \theta_{2}+\frac{\partial^{2} \mathbf{V}}{\partial \varepsilon_{i j}^{(3)} \partial \theta_{2}} \varepsilon_{i j}^{(3)} \theta_{2} \\
& +O^{3}\left(\varepsilon_{i j}^{(1)}, \theta_{2}, \varepsilon_{i j}^{(3)}\right) .
\end{aligned}
$$

We may define a fourth-order tensor of elastic coefficients for the solid (indice 1) and for ice (indice 3) and an elastic coefficient for water (indice 2):

$$
C_{i j k l}^{(1)}=\frac{\partial^{2} \mathbf{V}}{\partial \varepsilon_{i j}^{(1)} \partial \varepsilon_{k l}^{(1)}}, \quad C_{i j k l}^{(3)}=\frac{\partial^{2} \mathbf{v}}{\partial \varepsilon_{i j}^{(3)} \partial \varepsilon_{k l}^{(3)}} \quad \text { and } K_{2}=\frac{\partial^{2} \mathbf{v}}{\partial \theta_{2}^{2}}
$$

Two crossed terms appear in the development defining two second-order tensors of elastic coupling coefficients:

$$
C_{i j}^{s \omega}=\frac{\partial^{2} \mathbf{V}}{\partial \varepsilon_{i j}^{(1)} \partial \theta_{2}} \text { and } C_{i j}^{i \omega}=\frac{\partial^{2} \mathbf{V}}{\partial \varepsilon_{i j}^{(3)} \partial \theta_{2}} .
$$

These crossed terms describe, respectively, the exchange of elastic energy between solid and water and between ice and water. As an elastic wave propagates, the effective phases are submitted to the same macroscopic stresses but the resulting deformations of each phase are different since their rigidities are different. However since the effective phases fill the same space at macroscopic scale the deformation of one phase can be transmitted to another one. This phenomenon designated as elastic coupling will tend to be higher in a medium with a high specific area because it depends on the total area separating the phases. The crossed term relating the deformations of solid and of ice is missing in Eq. (1) according to the assumption on the absence of direct mechanical contact between these phases.

\section{Simplification for isotropic phases}

Disregarding the elastic coupling and using the definitions (2), the energy contribution of solid and ice in expression (1) are, respectively, given by

$$
\mathbf{V}_{1}=\frac{1}{2} C_{i j k l}^{(1)} \varepsilon_{i j}^{(1)} \varepsilon_{k l}^{(1)} \text { and } \mathbf{V}_{3}=\frac{1}{2} C_{i j k l}^{(3)} \varepsilon_{i j}^{(3)} \varepsilon_{k l}^{(3)} \text {. }
$$

The phases being statistically isotropic $C_{i j k l}^{(1)}$ and $C_{i j k l}^{(3)}$ can be expressed with only two independent coefficients and the expression of $\mathbf{V}_{1}$ and $\mathbf{V}_{3}$ can be simplified as

$$
\mathbf{V}_{1}=\mu_{1} d_{1}^{2}+\frac{1}{2} K_{1} \theta_{1}^{2} \text { and } \mathbf{V}_{3}=\frac{1}{2} K_{3} \theta_{3}^{2}+\mu_{3} d_{3}^{2} \text {, }
$$

where $K_{1}$ and $\mu_{1}$ are, respectively, the bulk and shear moduli of the effective solid. Here, $d_{1}$ and $\theta_{1}$ are the invariants of the strain tensor and are called the deviator and the dilatation (with $d_{i j}^{(1)} \cdot d_{i j}^{(1)}=d_{1}^{2}$ and $\varepsilon_{i i}^{(1)} \cdot \varepsilon_{j j}^{(1)}=\theta_{1}^{2}$ ). Analogous parameters are defined for ice. The phases being isotropic and water with a zero shear modulus it can be shown that the tensors of coupling coefficients can be reduced to two coefficients: one noted $C_{12}$ for the solid-water elastic coupling and the other $C_{23}$ for the ice-water elastic coupling. Finally the potential energy density is expressed as

$$
\begin{aligned}
\mathbf{V}= & \mu_{1} d_{1}^{2}+\frac{1}{2} K_{1} \theta_{1}^{2}+C_{12} \theta_{1} \theta_{2}+\frac{1}{2} K_{2} \theta_{2}^{2}+C_{23} \theta_{2} \theta_{3} \\
& +\frac{1}{2} K_{3} \theta_{3}^{2}+\mu_{3} d_{3}^{2} .
\end{aligned}
$$

\section{Stress-strain relationships}

For infinitesimal deformations the variation of potential energy may be expressed by a differential form:

$$
d \mathbf{V}=\frac{\partial \mathbf{V}}{\partial \varepsilon_{i j}^{(1)}} d \varepsilon_{i j}^{(1)}+\frac{\partial \mathbf{V}}{\partial \theta_{2}} d \theta_{2}+\frac{\partial \mathbf{V}}{\partial \varepsilon_{i j}^{(3)}} d \varepsilon_{i j}^{(3)}
$$

This expression allows the definition of a stress tensor for each phase:

$$
\sigma_{i j}^{(1)}=\frac{\partial \mathbf{V}}{\partial \varepsilon_{i j}^{(1)}}, \quad \sigma^{(2)}=\frac{\partial \mathbf{V}}{\partial \theta_{2}} \quad \text { and } \quad \sigma_{i j}^{(3)}=\frac{\partial \mathbf{V}}{\partial \varepsilon_{i j}^{(3)}} .
$$

Using the definitions (6) and the expression (4) of potential energy, the stress-strain relationships are expressed as

$$
\begin{aligned}
& \sigma_{i j}^{(1)}=2 \mu_{1} d_{i j}^{(1)}+\left(K_{1} \theta_{1}+C_{12} \theta_{2}\right) \delta_{i j}, \\
& \sigma^{(2)}=C_{12} \theta_{1}+K_{2} \theta_{2}+C_{23} \theta_{3}, \\
& \sigma_{i j}^{(3)}=2 \mu_{3} d_{i j}^{(3)}+\left(C_{23} \theta_{2}+K_{3} \theta_{3}\right) \delta_{i j},
\end{aligned}
$$

where $\delta_{i j}$ is the Kronecker's symbol. It may be noticed that the deformations of solid and of ice do not appear simultaneously in Eqs. (7a) and (7c) according to the assumption of absence of direct contact between these two phases. The elastic coefficients appearing in expressions (4) and (7) will be discussed later. For the present, let us only remark that $K_{1}, K_{2}$, and $K_{3}$ are the bulk moduli; $\mu_{1}$ and $\mu_{3}$ the shear moduli; $C_{12}$ and $C_{23}$ the coupling coefficients of effective solid (1), of unfrozen water as an effective fluid (2), and of ice as a second effective solid (3).

\section{Kinetic energy density $C$}

The displacements of solid, water, and ice are, respectively, represented by the vectors $u_{1}, u_{2}$, and $u_{3}$. The kinetic energy density $\mathbf{C}\left(\dot{u}_{1}, \dot{u}_{2}, \dot{u}_{3}\right)$ is a function of local velocities $\dot{u}_{1}, \dot{u}_{2}$, and $\dot{u}_{3}$ given by the time derivative of displacements. With a development of $\mathbf{C}$ to the second order and assuming that the phases motions are only caused by the wave propagation

$$
\mathrm{C}=\frac{1}{2} \rho_{11} \dot{u}_{1}^{2}+\rho_{12} \dot{u}_{1} \dot{u}_{2}+\frac{1}{2} \rho_{22} \dot{u}_{2}^{2}+\rho_{23} \dot{u}_{2} \dot{u}_{3}+\frac{1}{2} \rho_{33} \dot{u}_{3}^{2},
$$

where $\rho_{11}=\partial^{2} \mathrm{C} / \partial \dot{u}_{1}^{2}, \rho_{12}=\rho_{21}=\partial^{2} \mathrm{C} / \partial \dot{u}_{1} \partial \dot{u}_{2}, \rho_{22}=\partial^{2} \mathrm{C} / \partial \dot{u}_{2}^{2}$, $\rho_{23}=\rho_{32}=\partial^{2} \mathbf{C} / \partial \dot{u}_{2} \partial \dot{u}_{3}$, and $\rho_{33}=\partial^{2} \mathbf{C} / \partial \dot{u}_{3}^{2}$ are the elements of an induced mass tensor (Landau and Lifchitz, 1969). The crossed terms characterize the transfer of kinetic energy between solid and water and between ice and water. When an elastic wave propagates, local displacements of matter occur. In a porous medium the phases tend to have local velocities different from one another since sinuous fluid flows may occur in pores. Phases with different mass densities filling the same space at macroscopic scale implies exchanges of momentum between them. In fact the motion of one phase may induce the motion of another one by inertial effect. This 
phenomenon designated as inertial coupling becomes more important as the tortuosity of the medium increases. Here again, the crossed term in $\dot{u}_{1} \dot{u}_{3}$ has been rejected since there is no contact between solid and ice.

\section{Momenta}

The variation of kinetic energy may be expressed by the differential form:

$$
d \mathbf{C}=\frac{\partial \mathbf{C}}{\partial \dot{u}_{1}} d \dot{u}_{1}+\frac{\partial \mathrm{C}}{\partial \dot{u}_{2}} d \dot{u}_{2}+\frac{\partial \mathbf{C}}{\partial \dot{u}_{3}} d \dot{u}_{3} .
$$

Three generalized momenta are defined in this expression:

$$
\begin{aligned}
& \pi_{1}=\frac{\partial \mathbf{C}}{\partial \dot{u}_{1}}=\rho_{11} \dot{u}_{1}+\rho_{12} \dot{u}_{2}, \\
& \pi_{2}=\frac{\partial \mathbf{C}}{\partial \dot{u}_{2}}=\rho_{12} \dot{u}_{1}+\rho_{22} \dot{u}_{2}+\rho_{23} \dot{u}_{3}, \\
& \pi_{3}=\frac{\partial \mathbf{C}}{\partial \dot{u}_{3}}=\rho_{23} \dot{u}_{2}+\rho_{33} \dot{u}_{3} .
\end{aligned}
$$

Here, $\rho_{11}, \rho_{22}$, and $\rho_{33}$ are, respectively, the mass density of the effective solid, of water as effective fluid and of ice as a second effective solid; $\rho_{12}$ and $\rho_{23}$ are the mass densities induced by inertial coupling between solid and water and between ice and water. All these coefficients are the elements of the mass tensor and will be discussed later.

\section{Dissipation potential D}

Only dissipations caused by viscous flows are considered. The internal thermoconduction phenomena in solid or the frictions between solid grains are not studied and the losses caused by the presence of microbubbles of air are also neglected. The medium is assumed to be totally saturated.

\section{Viscous dissipations in frozen porous media}

The dissipation forces are assumed to be linear functions of velocities $\dot{u}_{1}, \dot{u}_{2}$, and $\dot{u}_{3}$ of the three phases or more precisely, of their differences. Hence, these forces are derived from a potential $\mathbf{D}$ given by the quadratic form:

$$
\begin{aligned}
\mathbf{D}= & \frac{1}{2}\left(\dot{u}_{1}-\dot{u}_{2}, \dot{u}_{1}-\dot{u}_{3}, \dot{u}_{2}-\dot{u}_{3}\right) \\
& \times\left(\begin{array}{lll}
\beta_{11} & \beta_{12} & \beta_{13} \\
\beta_{21} & \beta_{22} & \beta_{23} \\
\beta_{31} & \beta_{32} & \beta_{33}
\end{array}\right)\left(\begin{array}{l}
\left(\dot{u}_{1}-\dot{u}_{2}\right)^{*} \\
\left(\dot{u}_{1}-\dot{u}_{3}\right)^{*} \\
\left(\dot{u}_{2}-\dot{u}_{3}\right)^{*}
\end{array}\right) .
\end{aligned}
$$

where the asterisk denotes the complex conjugate. The coefficients $\beta_{i j}$ of the terms containing the difference $\dot{u}_{1}-\dot{u}_{3}$ are equal to zero in the expression of $\mathbf{D}$ since we have made the assumption of the absence of direct contact between solid and ice. The dissipation potential is reduced to:

$$
\mathbf{D}=\frac{1}{2}\left(\dot{u}_{1}-\dot{u}_{2}, \dot{u}_{2}-\dot{u}_{3}\right)\left(\begin{array}{ll}
\beta_{11} & \beta_{13} \\
\beta_{31} & \beta_{33}
\end{array}\right)\left(\begin{array}{c}
\left(\dot{u}_{1}-\dot{u}_{2}\right)^{*} \\
\left(\dot{u}_{2}-\dot{u}_{3}\right)^{*}
\end{array}\right) .
$$

D can be written in a simple form in a new system of coordinates diagonalizing the matrix of coefficients $\beta_{i j}$. The differences $\left(\dot{u}_{1}-\dot{u}_{2}\right)$ and $\left(\dot{u}_{2}-\dot{u}_{3}\right)$ are not affected by this linear transformation. With the condition $\beta_{11} \beta_{33}-\beta_{13} \beta_{31} \geqslant 0$ for
D to be positive, the dissipation potential is written as the superposition of two contributions:

$$
\mathbf{D}=\frac{1}{2} b_{11}\left|\dot{u}_{1}-\dot{u}_{2}\right|^{2}+\frac{1}{2} b_{33}\left|\dot{u}_{2}-\dot{u}_{3}\right|^{2},
$$

where $b_{11}$ and $b_{33}$ are real coefficients of a diagonal matrix and may be called, respectively, the friction coefficients between solid and water and between ice and water.

\section{E. Equations of motion}

The movements of a conservative mechanical system of $N$ degrees of freedom can be described with Lagrange's equations deduced from Hamilton's least-action principle. These equations initially established for a system of material points can be extended to continuous media (see Goldstein, 1980). For a conservative continuous system described by $N$ generalized coordinates $q_{i}$ of the three space variables $x_{j}$ and of time $t$, the $N$ Lagrange's equations are written as:

$$
\begin{aligned}
\frac{d}{d t} \frac{\partial \mathbf{L}}{\partial \dot{q}_{i}}+\sum_{j=1}^{3} \frac{d}{d x_{j}}\left[\frac{\partial \mathbf{L}}{\partial\left(\partial q_{i} / \partial x_{j}\right)}\right]-\frac{\partial \mathbf{L}}{\partial q_{i}}= & \\
& i=\{1,2, \ldots, N\},
\end{aligned}
$$

where $\mathbf{L}=\mathbf{C}-\mathbf{V}$ is the Lagrangian density per unit volume of continuous material.

For a nonconservative system, if the dissipation forces $f_{d}$ are derived from a potential $\mathbf{V}_{d}$ such that they verify the condition (see Arnold, 1974)

$$
f_{d}=-\frac{\partial \mathbf{V}_{d}}{\partial q_{i}}
$$

with

$$
\frac{\partial \mathbf{V}_{d}}{\partial q_{i}}=\frac{\partial \mathbf{D}}{\partial \dot{q}_{i}},
$$

the Lagrangian density can be expressed as for a conservative system

$$
\mathbf{L}=\mathbf{C}-\left(\mathbf{V}+\mathbf{V}_{d}\right)
$$

and Lagrange's equations are modified including the dissipation potential D:

$$
\frac{d}{d t} \frac{\partial \mathbf{L}}{\partial \dot{q}_{i}}+\sum_{j=1}^{3} \frac{d}{d x_{j}}\left[\frac{\partial \mathbf{L}}{\partial\left(\partial q_{i} / \partial x_{j}\right)}\right]-\frac{\partial \mathbf{L}}{\partial q_{i}}+\frac{\partial \mathbf{D}}{\partial \dot{q}_{i}}=0 .
$$

In our problem, three generalized coordinates are used and identified to the local displacements of the effective phases: $q_{1} \equiv u_{1}, q_{2} \equiv u_{2}$, and $q_{3} \equiv u_{3}$. The equations of motion are deduced using Lagrange's equations. They take a simple form using the expressions of stress tensors (7) and of generalized momenta (10):

$$
\begin{aligned}
& \sigma_{i j, j}^{(1)}=\dot{\pi}_{1}+b_{11}\left(\dot{u}_{2}-\dot{u}_{1}\right), \\
& \sigma_{, j}^{(2)}=\dot{\pi}_{2}-b_{11}\left(\dot{u}_{2}-\dot{u}_{1}\right)-b_{33}\left(\dot{u}_{2}-\dot{u}_{3}\right), \\
& \sigma_{i j, j}^{(3)}=\dot{\pi}_{3}+b_{33}\left(\dot{u}_{2}-\dot{u}_{3}\right) .
\end{aligned}
$$

The equations of movement are written in a matrix form by substituting the expressions (7) and (10) in (13): 


$$
\tilde{R} \operatorname{grad} \operatorname{div} \tilde{u}-\bar{\mu} \operatorname{curl} \operatorname{curl} \bar{u}=\bar{\rho} \tilde{u}+\tilde{A} \tilde{u},
$$

where the notation $\tilde{X}$ designates the matrix associated to the parameter $X$. Matrices of local displacements $\tilde{u}$, of velocities $\tilde{\tilde{u}}$ and of accelerations $\tilde{u}$ are used. The matrices of mass densities and friction coefficients are given by

$$
\tilde{\rho}=\left(\begin{array}{ccc}
\rho_{11} & \rho_{12} & 0 \\
\rho_{12} & \rho_{22} & \rho_{23} \\
0 & \rho_{23} & \rho_{33}
\end{array}\right)
$$

and

$$
\tilde{A}=\left(\begin{array}{ccc}
b_{11} & -b_{11} & 0 \\
-b_{11} & b_{11}+b_{33} & -b_{33} \\
0 & -b_{33} & b_{33}
\end{array}\right) \text {. }
$$

Matrices of rigidity and shear moduli are also used:

$$
\bar{R}=\left(\begin{array}{ccc}
R_{11} & R_{12} & 0 \\
R_{12} & R_{22} & R_{23} \\
0 & R_{23} & R_{33}
\end{array}\right)
$$

and

$$
\tilde{\mu}=\left(\begin{array}{ccc}
\mu_{11} & 0 & 0 \\
0 & 0 & 0 \\
0 & 0 & \mu_{33}
\end{array}\right)
$$

whose elements are given by

$$
\begin{aligned}
& R_{11}=K_{1}+\frac{4}{3} \mu_{1}, \quad R_{12}=R_{21}=C_{12}, \quad R_{22}=K_{2}, \\
& R_{23}=R_{32}=C_{23}, \quad R_{33}=K_{3}+\frac{4}{3} \mu_{3}, \\
& \mu_{11}=\mu_{1}, \mu_{33}=\mu_{3} .
\end{aligned}
$$

\section{F. Equations of propagation}

As a movement can always be decomposed in translational and rotational parts, the displacement vectors $u_{1}, u_{2}$, and $u_{3}$ can be written, respectively, as the sum of two independent vectors. An irrotational vector corresponding to translations of matter without shear deformations and a vector without divergence describing rotations without volume change. Thus the displacement vector $u_{i}$ of the phase indiced $i$ is derived from a scalar potential $\varphi_{i}$ and a vector potential $\psi_{i}$ :

$$
u_{i}=u_{L i}+u_{T i}
$$

with

$$
u_{L i}=\operatorname{grad} \varphi_{i}
$$

and

$$
u_{T i}=\operatorname{curl} \psi_{i} .
$$

Applying the divergence operator to each member of the equations of movement (14) provides the equations of propagation for longitudinal waves characterized by displacements of matter colinear to the direction of propagation:

$$
\tilde{R} \nabla^{2} \tilde{\varphi}=\tilde{\rho} \tilde{\varphi}+\tilde{A} \tilde{\varphi}
$$

where $\nabla^{2}$ represents the Laplace operator. In a symmetrical way, applying the rotational operator to each member of the equations of movement (14) provides the equations of propagation for transverse waves characterized by displacements of matter perpendicular to the direction of propagation:

$$
\bar{\mu} \nabla^{2} \tilde{\psi}=\bar{\rho} \bar{\psi}+\tilde{A} \overline{\dot{\psi}}
$$

Before going further in the problem we examine two limit cases: If the medium is totally defrosted the conditions $\phi_{w} \rightarrow \varepsilon$ and $\phi_{i} \rightarrow 0$ are satisfied. The parameters indiced 3 will vanish and the equations reduce to the equations of propagation given by Biot for porous media containing only a solid and a fluid. On the contrary, if the medium is totally frozen one may study the limit: $\phi_{w} \rightarrow 0$ and $\phi_{i} \rightarrow \varepsilon$. The parameters indiced 2 will vanish and the equations of propagation can be written with matrices of the second order. This limit case may be seen as a model of acoustic propagation in heterogeneous media composed of two solids. This model would be different from other formulations by the prediction of several longitudinal and transverse waves.

\section{G. Resolution of the equations of propagation}

The mass density matrix can be inverted if it is regular. Then three eigenvalues are provided from diagonalization of $\tilde{\rho}^{-1} \tilde{R}$ and two from diagonalization of $\tilde{\rho}^{-1} \tilde{\mu}$. These eigenvalues may give the velocity and attenuation of three longitudinal and two transverse waves. Although natural, this method needs the calculation of many determinants and expressions. Another method may be used consisting in a linear transformation of coordinates in order that the kinetic energy $\mathbf{C}$ and potential energy $\mathbf{V}$ are reduced simultaneously to a sum of square terms.

\section{Longitudinal solutions}

In a first step let us examine the case without attenuation disregarding the matrix $\tilde{A}$ of friction coefficients. If rigidity and mass density matrices are symmetrical and with real coefficients and if the conditions for the kinetic and potential energies to be positive definite forms have been previously determined by diagonalizing $\tilde{\rho}$ and $\tilde{R}$ separately, one may find a system of coordinates $Q_{i}\{i=1,2,3\}$ in which (see Arnold, 1974):

$$
\mathbf{C}=\frac{1}{2} \dot{Q}_{i}^{2} \text { and } \mathbf{V}=\frac{1}{2} \Lambda_{i} Q_{i}^{2} .
$$

In this system, the equations of propagation are for longitudinal solutions:

$$
Q_{i}=\operatorname{grad} \Phi_{i}, \quad \Delta \Phi_{i}-\Lambda_{i} \ddot{\boldsymbol{\Phi}}=0,
$$

where the coefficients $\Lambda_{i}$ verify

$$
\left|\bar{\rho}-\Lambda_{i} \tilde{R}\right|=0 \text {. }
$$

The coefficients $\Lambda_{i}$ are called the eigenvalues of $\bar{\rho}$ relatively to $\tilde{R}$. A difficulty appears for continuous media where the potential energy is expressed as a function of the invariants of strain tensors [see Eq. (4)] and not directly as a function of displacements. Nevertheless, for sinusoidal displacements in space and time the dilatations $\theta_{i}$ and the accelerations are linear functions of the displacements $u_{i}$ : 


$$
\theta_{i}=\operatorname{div} u_{i}=j k \cdot u_{i} \text { and } \ddot{u}_{i}=-\omega^{2} u_{i} .
$$

Here, $j$ is the imaginary unit complex number $\left(j^{2}=-1\right), k$ is the wave number, and $\omega$ the angular frequency. Thus the potential energy can be expressed as a function of displacements as required and the method is usable. The condition (18) leads to a third degree characteristic equation:

$$
\begin{aligned}
& \Lambda^{3} \operatorname{det} \tilde{R}-\Lambda^{2}\left[\left(\rho_{11} \operatorname{det} \tilde{R}_{i w}+\rho_{22} \operatorname{det} \tilde{R}_{s i}+\rho_{33} \operatorname{det} \tilde{R}_{s w}\right)\right. \\
& \left.\quad-2\left(R_{11} R_{23} \rho_{23}+R_{33} R_{12} \rho_{12}\right)\right] \\
& \quad+\Lambda\left[\left(R_{11} \operatorname{det} \tilde{\rho}_{i w}+R_{22} \operatorname{det} \bar{\rho}_{s i}+R_{33} \operatorname{det} \bar{\rho}_{s w}\right)\right. \\
& \left.\quad-2\left(\rho_{11} \rho_{23} R_{23}+\rho_{33} \rho_{12} R_{12}\right)\right]-\operatorname{det} \bar{\rho}=0
\end{aligned}
$$

with

$$
\begin{aligned}
& \operatorname{det} \tilde{R}_{s w}=R_{11} R_{22}-R_{12}^{2}, \\
& \operatorname{det} \tilde{R}_{i w}=R_{22} R_{33}-R_{23}^{2}, \\
& \operatorname{det} \tilde{R}_{s i}=R_{11} R_{33}, \\
& \operatorname{det} \tilde{R}=R_{11} R_{22} R_{33}-R_{23}^{2} R_{11}-R_{12}^{2} R_{33},
\end{aligned}
$$

and

$$
\begin{aligned}
& \operatorname{det} \bar{\rho}_{s w}=\rho_{11} \rho_{22}-\rho_{12}^{2}, \\
& \operatorname{det} \bar{\rho}_{i w}=\rho_{22} \rho_{33}-\rho_{23}^{2}, \\
& \operatorname{det} \tilde{\rho}_{s i}=\rho_{11} \rho_{33}, \\
& \operatorname{det} \bar{\rho}=\rho_{11} \rho_{22} \rho_{33}-\rho_{23}^{2} \rho_{11}-\rho_{12}^{2} \rho_{33} .
\end{aligned}
$$

The longitudinal wave velocities are given by the relationship: $\Lambda=k^{2} / \omega^{2}=1 / V_{L}^{2}$. The notation det $\bar{X}$ designates the determinant of the matrix $\bar{X}$. The symmetry of the roles played by the matrices of rigidity and of mass density in Eq. (19) may be noticed.

Now, we will study the propagation with attenuation by introducing $\tilde{A}$ in the formulation. A linear relationship exists between local velocities and accelerations for time sinusoidal displacements:

$$
\dot{\varphi}_{i}=-(j / \omega) \ddot{\varphi}_{i} .
$$

Thus the complex term $j^{A_{i j} / \omega}$ must be subtracted from each element $\rho_{i j}$ of the mass density matrix in the characteristic Eq. (19). Hence a new characteristic equation is obtained with complex coefficients:

$$
Z_{3} \Lambda^{3}-Z_{2} \Lambda^{2}+Z_{1} \Lambda-Z_{0}=0+0 j .
$$

The three complex solutions:

$$
\Lambda_{1}^{1 / 2}=R_{1}+j I_{1}, \quad \Lambda_{2}^{1 / 2}=R_{2}+j I_{2}
$$

and

$$
\Lambda_{3}^{1 / 2}=R_{3}+j I_{3}
$$

provide three longitudinal wave velocities:

$$
V_{L 1}=1 /\left|R_{1}\right|, \quad V_{L 2}=1 /\left|R_{2}\right|, \quad V_{L 3}=1 /\left|R_{3}\right|,
$$

and the three corresponding attenuation coefficients:

$$
\alpha_{L 1}=\omega / I_{1}, \quad \alpha_{L 2}=\omega / I_{2}, \quad \alpha_{L 3}=\omega / I_{3} .
$$

The indices 1, 2, 3 used here refer to solutions of the wave equation and not to the material effective phases (solid, water, and ice) used elsewhere in this paper.

\section{Transverse solutions}

An analogous method to the one for longitudinal waves may be used. In fact we just have to replace the rigidity matrix $\tilde{R}$ by the shear moduli matrix $\tilde{\mu}$ in the equations. Disregarding the matrix $\tilde{A}$, the characteristic equation for transverse waves is written as:

$$
\begin{array}{r}
\Lambda^{2} \rho_{22} \operatorname{det} \tilde{\mu}_{s i}-\Lambda\left(\mu_{11} \operatorname{det} \tilde{\rho}_{i w}+\mu_{33} \operatorname{det} \tilde{\rho}_{\text {sw }}\right)+\operatorname{det} \tilde{\rho}=0 \\
\text { with } \operatorname{det} \tilde{\mu}_{s i}=\mu_{11} \mu_{33} .
\end{array}
$$

Replacing $\rho_{i j}$ by $\left(\rho_{i j}-j^{A} i j / \omega\right)$ in (21) leads to the resolution of a second-degree characteristic equation with complex coefficients. The solutions being:

$$
\Lambda_{1}^{1 / 2}=R_{1}+j I_{1} \text { and } \Lambda_{2}^{1 / 2}=R_{2}+j I_{2},
$$

the velocities of transverse waves are given by:

$$
V_{T 1}=1 /\left|R_{1}\right| \text { and } V_{T 2}=1 /\left|R_{2}\right|,
$$

and the attenuation coefficients by:

$$
\alpha_{T 1}=\omega / I_{1} \text { and } \alpha_{T 2}=\omega / I_{2} \text {. }
$$

\section{DETERMINATION OF THE GENERALIZED ELASTIC COEFFICIENTS $\boldsymbol{R}_{\boldsymbol{\eta}}$}

In order to complete the model, it is necessary to express the coefficients $\boldsymbol{R}_{i j}$ appearing in Eqs. (4) and (7) with the help of known or measurable parameters. From conceptual experiments on the compressibility of fluid-saturated porous media, Biot and Willis (1957) have found expressions of the generalized coefficients as functions of the phase proportions, of the solid and fluid elastic moduli and of the solid frame stiffness. For frozen porous media, the ice parameters must be added to these parameters for the determination of the generalized elastic coefficients. The elastic moduli of the ice matrix will be studied later. In order to simplify the problem, the influence of the imposed external temperature on the solid, water, and ice elastic coefficients are neglected here but one must know that these coefficients may be significantly modified for large temperature variations (see Kittel, 1971 for example). The coefficients $R_{i j}$ may be deduced from a physical interpretation of Biot's generalized elastic coefficients. This method based on a physical argument appears as intuitive. A more mathematical one leading to the same results has been proposed by Leclaire (1992).

For a fluid-saturated porous medium (without ice), three generalized elastic coefficients $R_{i j}$ were defined in Biot's theory (see Johnson and Plona, 1982). We express them in a simple form as:

$$
\begin{aligned}
& R_{11}=\left[(1-c) \phi_{s}\right]^{2} K_{\mathrm{av}}+K_{\mathrm{sm}}+4 \mu_{\mathrm{sm}} / 3, \\
& R_{12}=\left[(1-c) \phi_{s}\right] \phi_{f} K_{\mathrm{av}}, \\
& R_{22}=\phi_{f}^{2} K_{\mathrm{av}},
\end{aligned}
$$

with 


$$
c=\frac{K_{\mathrm{sm}}}{\phi_{s} K_{s}}
$$

and

$$
K_{\mathrm{av}}=\left(\frac{(1-c) \phi_{s}}{K_{s}}+\frac{\phi_{f}}{K_{f}}\right)^{-1}
$$

where $\phi_{s}$ and $\phi_{f}$ are, respectively, the solid and fluid proportions, $K_{s}$ and $K_{f}$ the solid and fluid bulk moduli, and $K_{\mathrm{sm}}$ and $\mu_{\mathrm{sm}}$ the solid matrix bulk and shear moduli. As a parenthesis, the matrix rigidity must be distinguished from the solid rigidity. The first one concerns the solid frame and is defined at macroscopic scale only. The second characterizes the elasticity of the solid material that forms the solid matrix.

\section{A. Physical interpretation of Biot's elastic coefficients}

The coefficient $c$ is always between 0 and 1 . For a nonconsolidated medium such as a suspension of solid grains in a fluid, $K_{\mathrm{sm}}=\mu_{\mathrm{sm}}=0$ and $c=0$. If the solid matrix is consolidated, Hashin and Sthrikman (1963) have shown that for a proportion $\phi_{s}$ of solid, $K_{\mathrm{sm}}$ is always less than $\phi_{s} K_{s}$. This upper bound for $c=1$ would correspond to a situation where all the solids would arrange to form a monolithic block. The coefficient $c$ given by $c=K_{\mathrm{sm}} / \phi_{s} K_{s}$ may be called the bulk consolidation coefficient as it characterizes the degree of consolidation of the solid matrix.

In a first step let us study nonconsolidated media: $c=0$. $K_{\mathrm{av}}$ is given by the Hashin-Sthrikman's lower bound (1963): $K_{\mathrm{av}}=\left(\phi_{s} / K_{s}+\phi_{f} / K_{f}\right)^{-1}$. This expression may be compared to the expression giving the equivalent stiffness of two springs in a serial configuration. $K_{\mathrm{av}}$ may be designated as the "average" bulk modulus of the porous medium since it is given by the weighted average of the inverse of phases bulk moduli. Then Biot's elastic coefficients for nonconsolidated media are given by:

$$
R_{11}=\phi_{s}^{2} K_{\mathrm{av}}, \quad R_{12}=\phi_{s} \phi_{f} K_{\mathrm{av}}, \quad R_{22}=\phi_{f}^{2} K_{\mathrm{av}} .
$$

For the general case of a solid matrix with a nonzero modulus $K_{\mathrm{sm}}$, the consolidation coefficient $c$ plays a role and the average bulk modulus $K_{\mathrm{av}}$ is increased:

$$
K_{\mathrm{av}}=\left[(1-c) \phi_{s} / K_{s}+\phi_{f} / K_{f}\right]^{-1} .
$$

The quantity $(1-c)$ acts as a weighting factor in the expression of the average modulus including the solid matrix rigidity. $K_{\mathrm{av}}$ should be the coefficient evaluated by applying a pressure on the porous medium and measuring the induced deformation. In the general case, Biot's elastic coefficients are given by Eq. (22).

\section{B. Frozen media}

Let us now consider frozen porous media. We may define a bulk consolidation coefficient for the solid as $c_{1}=K_{\mathrm{sm}} / \phi_{s} K_{s}$ and for ice as $c_{3}=K_{\mathrm{im}} / \phi_{i} K_{i}$. An average bulk modulus may also be defined as

$$
K_{\mathrm{av}}=\left[\left(1-c_{1}\right) \phi_{s} / K_{s}+\phi_{w} / K_{w}+\left(1-c_{3}\right) \phi_{i} / K_{i}\right]^{-1} .
$$

The coefficients appearing in Eqs. (4) and (7) are deduced by a simple analogy with the coefficients given in Eq. (22):

$$
\begin{aligned}
& K_{1}=\left[\left(1-c_{1}\right) \phi_{s}\right]^{2} K_{\mathrm{av}}+K_{\mathrm{sm}}, \\
& C_{12}=\left[\left(1-c_{1}\right) \phi_{s}\right] \phi_{w} K_{\mathrm{av}}, \\
& K_{2}=\phi_{w}^{2} K_{\mathrm{av}}, \\
& C_{23}=\left[\left(1-c_{3}\right) \phi_{i}\right] \phi_{w} K_{\mathrm{av}}, \\
& K_{3}=\left[\left(1-c_{3}\right) \phi_{i}\right]^{2} K_{\mathrm{av}}+K_{\mathrm{im}},
\end{aligned}
$$

where $K_{s}, K_{w}$, and $K_{i}$ are, respectively, the bulk moduli of solid, water, and ice. $K_{\mathrm{sm}}$ and $K_{\mathrm{im}}$ are the bulk moduli of solid matrix and ice matrix. The generalized elastic coefficients $R_{i j}$ are given with the help of relations (15a).

\section{Shear moduli of frozen media}

In a frozen porous medium, solid and ice matrices are supposed to coexist without contact since they are separated by a film of unfrozen water. In such a situation, if the interstitial water had no viscosity, the shear deformations of the two matrices would be independent and the moduli $\mu_{1}$ and $\mu_{3}$ defined in Eqs. (4) and (7) would be, respectively, equal to the shear moduli of solid matrix and of ice matrix. Nevertheless, the unfrozen water layer can be very thin when the porous medium is almost totally frozen. In this situation the unfrozen water viscosity can play an important role for the transmission of shear deformations from one matrix to the other. The sludy of the oscillations of an unlimited plane surface along its own plane (Landau and Lifchitz, 1969 or Biot, 1956a) in a viscous fluid has shown that a shear wave may exist in the fluid and that its direction of propagation is perpendicular to the surface. The phase velocity of this wave is given by

$$
\omega / \operatorname{Re}(k)=\left(2 \omega \nu_{f}\right)^{1 / 2}=\left(2 \omega \eta_{f} / \rho_{f}\right)^{1 / 2},
$$

where $\omega$ is the angular frequency, $k$ the complex wave number, $\rho_{f}$ the fluid mass density, and $\nu_{f}$ its kinematic viscosity related to the dynamic viscosity by $\nu_{f}=\eta_{f} / \rho_{f}$. This wave is quickly attenuated in the fluid. The viscous skin depth $\delta$ which correspond to a decrease of the wave amplitude by a factor $\exp (1)$ is given by:

$$
\delta=\left(2 v_{f} / \omega\right)^{1 / 2} .
$$

The decrease of unfrozen water proportion in frozen porous media is associated with a decrease of the average water film thickness $h$ between the two matrices. When $h$ is of the order of the viscous skin depth, shear waves can be transmitted from one matrix to the other through unfrozen water. Looking at Eq. (24), things happen as if water had a dynamic shear modulus $2 \omega \eta_{w}$ at angular frequency $\omega, \eta_{w}$ being the dynamic viscosity of interstitial water. The moduli $\mu_{1}$ and $\mu_{3}$ defined in Eqs. (4) and (7) are given by expressions analogous to those of coefficients $K_{1}$ and $K_{3}$ :

$$
\begin{aligned}
& \mu_{1}=\left[\left(1-g_{1}\right) \phi_{\mathrm{s}}\right]^{2} \mu_{\mathrm{av}}+\mu_{\mathrm{sm}}, \\
& \mu_{3}=\left[\left(1-g_{3}\right) \phi_{i}\right]^{2} \mu_{\mathrm{av}}+\mu_{\mathrm{im}},
\end{aligned}
$$

where a shear coefficient of consolidation is defined for solid matrix as $g_{1}=\mu_{\mathrm{sm}} / \phi_{s} \mu_{s}$ and for ice matrix as $g_{3}=\mu_{\mathrm{im}} / \phi_{i} \mu_{i}$. An average shear modulus including the effect of water viscosity is also defined for frozen porous media as 


$$
\mu_{\mathrm{av}}=\left[\left(1-g_{1}\right) \phi_{s} / \mu_{s}+\phi_{w} / 2 \omega \eta_{w}+\left(1-g_{3}\right) \phi_{i} / \mu_{i}\right]^{-1},
$$

where $\mu_{s}$ and $\mu_{i}$ are, respectively, the solid and ice shear moduli; $\mu_{\mathrm{sm}}$ and $\mu_{\mathrm{im}}$ the shear moduli of solid and ice matrices.

It may be noticed that only the real part of the complex wave number is considered in Eq. (24). The imaginary part might play a role in the wave attenuation when the unfrozen water thickness is on the order of the viscous skin depth but this effect is neglected in the present study. We may also remark that in a rigorous way coefficients $\mu_{12}, \mu_{23}$, and $\mu_{2}$ should also be defined in Eqs. (4) and (7) since water has been considered to have a shear modulus. However, these coefficients given by $\mu_{12}=\left[\left(1-g_{1}\right) \phi_{s}\right] \phi_{w} \mu_{\mathrm{av}}$, $\mu_{23}=\left[\left(1-g_{3}\right) \phi_{i}\right] \phi_{w} \mu_{\mathrm{av}}$ and $\mu_{2}=\phi_{w}^{2} \mu_{\mathrm{av}}$ can be neglected with respect to $\mu_{1}$ and $\mu_{3}$. Indeed it will be shown that $\mu_{\mathrm{av}}$ is small when $\phi_{w}$ tends toward porosity $\varepsilon$ and inversely, an increase of $\mu_{\mathrm{av}}$ correspond to a decrease of $\phi_{w}$ toward 0 . Thus $\mu_{12}, \mu_{23}$, and $\mu_{2}$ can be neglected since the factor $\phi_{w} \mu_{\mathrm{av}}$ acts in their expressions.

Let us study the variations of the average shear modulus $\mu_{\mathrm{av}}$ as a function of water proportion $\phi_{w}$. With a solid shear modulus $\mu_{s}$ of the order of $10^{10} \mathrm{~Pa}$, a viscosity of the order of $10^{-3} \mathrm{~kg} \mathrm{~m}^{-1} \mathrm{~s}^{-1}\left(\eta_{w}=1.798 \times 10^{-3} \mathrm{~kg} \mathrm{~m}^{-1} \mathrm{~s}^{-1}\right.$ at $\left.0^{\circ} \mathrm{C}\right)$, the wave frequency given by $f=\omega / 2 \pi$ should be in the order of $10^{12} \mathrm{~Hz}$ for dynamic shear modulus $2 \omega \eta_{e}$ of water to be comparable to the solid shear modulus. Of course, this frequency value is out of the range of our study. The frequencies actually used are less than $10^{6} \mathrm{~Hz}$ and as a consequence the quantity $\phi_{w} / 2 \omega \eta_{w}$ is much greater than $\left(1-g_{1}\right) \phi_{s} / \mu_{s}$ and $\left(1-g_{3}\right) \phi_{i} / \mu_{i}$. Thus $\mu_{\mathrm{av}}$ is much smaller than $\mu_{\mathrm{sm}}$ and $\mu_{\text {im }}$ except when the unfrozen water proportion $\phi_{w}$ tends toward 0 . In that situation where the unfrozen water layer is very thin, the viscosity effects are important and $\mu_{\mathrm{av}}$ can be of the order of $\mu_{\mathrm{sm}}$ and $\mu_{\mathrm{im}}$.

\section{High-frequency range}

From the study of the motion of a fluid oscillating in a two- or three-dimensional capillary pore, Biot (1956b) has defined a characteristic frequency depending on the pore radius $r_{p}$ and identified two regimes of flow: a low-frequency regime where the friction forces vary as the square of frequency and a higher frequency regime where the friction forces vary as the square root of frequency. Referring to Biot's results, this problem is formulated by replacing the static viscosity $\eta_{w}$ of water by a dynamical viscosity $\eta_{w} F(\xi)$ where frequency acts through the variable

$$
\xi=h / 2\left(\omega \rho_{f} / \eta_{f}\right)^{1 / 2} .
$$

Here, $h$ is the average thickness of the unfrozen water layer playing the role of the pore diameter and may be evaluated as a function of the average radius $r_{s}$ of solid grains as (see Leclaire, 1992):

$$
h=r_{s}\left[\left(1+\phi_{w^{*}} / \phi_{s}\right)^{1 / 3}-1\right] .
$$

The complex correction function for viscosity $F(\xi)$ (see Biot, 1956b) will be modeled numerically by

$$
\begin{aligned}
\operatorname{Re}[F(\xi)]= & 1+(1 / 0.7178) \cdot\left[e^{+0.7178(\xi-3.2)}\right] / 12, \\
& \text { if } \xi \leqslant 3.2 \\
\operatorname{Re}[F(\xi)]= & 1 / 2+\left[2 \xi+e^{-0.7178(\xi-3.2)}\right] / 12 \\
& \text { if } \xi \geqslant 3.2
\end{aligned}
$$

and

$$
\operatorname{Im}[F(\xi)]=\xi / 6 .
$$

As an assumption in the previous development, the properties of unfrozen water are supposed to be those of free water. Water viscosity is a function of temperature. Gilpin (1980) has given a polynomial regression formula usable only between -10 and $+10^{\circ} \mathrm{C}$. For our problem an exponential regression formula has been preferred. This formula obtained from experimental data on free-water viscosity between 0 and $-10^{\circ} \mathrm{C}$ (Handbook, 1965-1966) may be used at temperatures lower than $-10^{\circ} \mathrm{C}$ and is given by: $\eta_{w}(T)=1.798 .10^{-3} e^{-0,03753 . T}$.

Elsewhere, Peschel (1968) has shown that the viscosity of a film of water between two quartz plates increases and can take very high values as the distance between the two plates decreases. The viscosity may be evaluated as a function of the average thickness $h$ of unfrozen water layer by: $\eta_{w}(h) / \eta_{w}(\infty)=(450+h) / h$, where $h$ is expressed in angstroms. $\eta_{w}(\infty)$ is the viscosity of free water and $\eta_{w}(h)$ the viscosity of interstitial water.

\section{ELASTIC COEFFICIENTS OF HETEROGENEOUS MATERIALS}

The generalized elastic coefficients $R_{i j}$ have been expressed as functions of the phases' elastic coefficients. These are supposed to be known except for the moduli of the solid matrix and of ice matrix. As already mentioned, it is important to distinguish the elastic moduli of the solid materials from those of solid skeletal frames. Allow us to take the example of a steel spring fixed at one end. If a traction is applied on the other end the extension is in the linear regime proportional to the spring rigidity coefficient. Now let us imagine that one is able to stretch the spring until it takes the shape of a thread. If a traction of the same intensity as for the spring is applied on the thread, its extension, proportional to the inverse of Young's modulus of steel, will be much different from the extension of the spring. Thus a spring can be characterized with two elastic coefficients. One corresponds to the spring itself and the other to the material that forms it. The same distinction is valid for a porous medium between the solid material and the solid matrix. Several authors have studied the effective properties of heterogenous materials (Hill, 1965; Budiansky, 1965; Berryman, 1979; Kuster and Toksöz, 1974). The methods proposed by these authors may be used for the solid matrix if its elastic moduli are not attainable experimentally or for the ice matrix considered as a particular heterogeneous material containing only two phases: the ice substrate and air filling the apparent porosity $\left(\phi_{s}+\phi_{w}\right)$. Since they are generally valid for low porosities only, these methods have been rejected except for the limit case of a totally frozen medium where the apparent porosity 
of the ice matrix is equal to $\phi_{s}$. Furthermore, a transition of the ice matrix from a connected state to a disconnected or a granular state occurs during a thawing process as the bonds relating the ice nuclei disappear progressively. The classical models are unable to describe such a phenomenon and another method must be used.

\section{A. Percolation model}

The percolation theory can be used to describe the transition of a system or a material between the continuous state and the discontinuous state. During this process called transition of percolation, connections appear or disappear between the elements of the system or material. The induced modifications of the system configuration or material properties are governed by a power law independent of the system or material.

De Gennes (1976) has established a relationship between the electrical conductivity of a network and the elasticity of a polymeric gel during a transition of percolation. For our study, the ice matrix elastic moduli are evaluated as functions of ice proportion $\phi_{i}$ by

$$
\begin{gathered}
K_{\mathrm{im}}=K_{\max }\left[\left(\phi_{i}-\phi_{i \mathrm{Th}}\right) /\left(\phi_{i \max }-\phi_{i \mathrm{Th}}\right)\right]^{\tau}, \\
\mu_{\mathrm{im}}=\mu_{\text {max }}\left[\left(\phi_{i}-\phi_{i \mathrm{Th}}\right) /\left(\phi_{i \max }-\phi_{i \mathrm{Th}}\right)\right]^{\tau} .
\end{gathered}
$$

These expressions may be used only if $\phi_{i}$ is higher than $\phi_{i \mathrm{Th}}$ and less than a maximum proportion $\phi_{i \text { max }}$ corresponding to the beginning of the transition of percolation. $K_{\mathrm{im}}$ and $\mu_{\text {im }}$ take, respectively, the particular values $K_{\max }$ and $\mu_{\max }$ for $\phi_{i}=\phi_{i \text { max }}$. Several assumptions have been made in order to evaluate the constants $\phi_{i \mathrm{Th}}, \phi_{i \max }, \tau, K_{\max }$ and $\mu_{\max }$.

(i) The same critical exponent is used for $K_{\mathrm{im}}$ and $\mu_{\mathrm{im}}$. For the elasticity of a tridimensional system the chosen value is $\tau=3.8$ (Deptuck et al., 1985).

(ii) $\phi_{i \max }=\varepsilon$ if the transition of percolation is supposed to begin as the thawing process begins.

(iii) For simplicity, $\phi_{i \mathrm{Th}}$ is supposed to be $\mathbf{0}$ assuming a nonzero matrix rigidity until the porous medium is totally defrosted.

(iv) $K_{\max }$ and $\mu_{\max }$ may be obtained from the classical models (Kuster-Toksöz's model) for $\phi_{i}=\varepsilon$. The elastic moduli of ice matrix calculated with the help of expressions (27) are represented as functions of apparent porosity in Fig. 2 .

\section{DETERMINATION OF THE GENERALIZED MASS DENSITIES $\rho_{i j}$}

The generalized mass densities $\rho_{i j}$ appearing in the expressions (8) of kinetic energy and (10) of generalized momenta may be expressed as functions of the phase proportions, of their mass densities and of two factors characterizing the geometrical aspect of solid and ice matrices. The method chosen for this purpose consists in calculating the kinetic energy density $\mathbf{C}$ in a pore. Then the coefficients $\rho_{i j}$ will be identified to those of the expression obtained for $\mathbf{C}$.

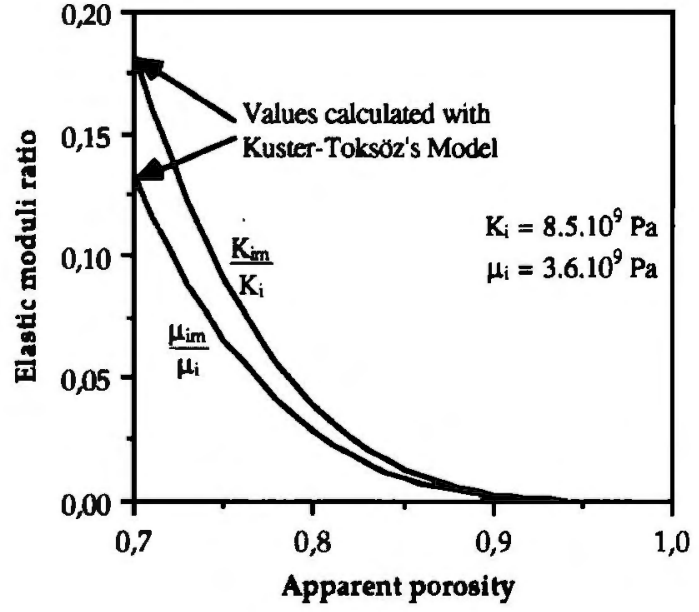

FIG. 2. Elastic moduli of the ice matrix calculated as functions of its apparent porosity.

\section{A. Kinetic energy density in a pore filled of water and containing an ice nucleus}

Biot (1962) has studied the kinetic energy of the unit volume of a compressible, fluid-saturated porous material. In the following development, a calculation of the kinetic energy density $\mathbf{C}$ is presented for a pore containing an ice nucleus surrounded by unfrozen water that may flow between solid and ice. The method used to calculate $\mathbf{C}$ consists in considering a particular case where no relative motion would exists between ice and water and a particular case where no relative motion would exists between solid and water. The kinetic energy is then calculated for each case using Biot's results and the global kinetic energy is deduced for the general case from a combination of these two particular cases. Let us first define two particular velocities:

$$
\dot{w}_{1}=\phi_{w}\left(\dot{u}_{2}-\dot{u}_{1}\right) \text { and } \dot{w}_{3}=\phi_{w}\left(\dot{u}_{2}-\dot{u}_{3}\right) \text {, }
$$

characterizing, respectively, water-solid flow and water-ice flow. For a Poiseuille's viscous flow, the relative velocity field between water and solid $v_{1}$ and between water and ice $v_{3}$ are expressed as

$$
v_{1}=\alpha_{i j}^{(1)}\left(\dot{w}_{j}\right)_{1} \text { and } v_{3}=\alpha_{i j}^{(3)}\left(\dot{w}_{j}\right)_{3}
$$

where the coefficients $\alpha_{i j}^{(1)}$ depend on the solid geometry and $\alpha_{i j}^{(3)}$ on ice geometry.

In a first step let us assume that no relative motion exists between ice and water. In this case $\dot{u}_{3}=\dot{u}_{2}, \dot{w}_{3}=0$ and $v_{3}=0$. The kinetic energy is then written as

$$
\mathbf{C}_{1}=\frac{1}{2} \rho_{1} \dot{u}_{1}^{2}+\frac{1}{2} \rho_{w} \iiint_{\Omega}\left(\dot{u}_{1}+v_{1}\right)^{2} d \Omega+\frac{1}{2} \rho_{3} \dot{u}_{2}^{2},
$$

with $\rho_{1}=\phi_{s} \rho_{s}, \rho_{2}=\phi_{w} \rho_{w}$, and $\rho_{3}=\phi_{i} \rho_{i}$. The integration is made on the volume $\Omega$ of water in the pore (i.e., the pore volume minus the ice nucleus volume). If $\left(\dot{u}_{1}+v_{1}\right)^{2}$ is expanded, the first term of the space integral is written as

$$
\frac{1}{2} \rho_{w} \iiint_{\Omega} \dot{u}_{1}^{2} d \Omega=\frac{1}{2} \rho_{2} \dot{u}_{1}^{2}
$$

and the other terms are given by 


$$
\rho_{w} \iiint_{\Omega} \dot{u}_{1} v_{1} d \Omega=\rho_{w} \dot{u}_{1} \iiint_{\Omega} v_{1} d \Omega=\rho_{w} \dot{u}_{1} \dot{w}_{1}
$$

and

$$
\rho_{w} \iiint_{\Omega} v_{1}^{2} d \Omega=\sum_{i j}\left(m_{i j}\right)_{1} \dot{w}_{i} \dot{w}_{i},
$$

with

$$
\left(m_{i j}\right)_{1}=\rho_{w} \iiint_{\Omega} \sum_{k} \alpha_{k i}^{(1)} \alpha_{k j}^{(1)} d \Omega=\left(m_{j i}\right)_{1} .
$$

With the assumption of statistical isotropy, the matrix $\left(m_{i j}\right)_{1}$ can be written in a simpler form using Kronecker's symbol $\delta_{i j}$ :

$$
\left(m_{i j}\right)_{1}=m_{1} \delta_{i j} .
$$

The kinetic energy is expressed as

$$
\mathrm{C}_{1}=\frac{1}{2}\left(\rho_{1}+\rho_{2}\right) \dot{u}_{1}^{2}+\rho_{w} \dot{u}_{1} \dot{w}_{1}+\frac{1}{2} m_{1} \dot{w}_{1}^{2}+\frac{1}{2} \rho_{3} \dot{u}_{2}^{2} .
$$

Now we will examine the case where no relative motion would exist between solid and water such that $\dot{u}_{1}=\dot{u}_{2}$. Using an analogous method as in the previous case, one may obtain the expression of kinetic energy

$$
\mathrm{C}_{3}=\frac{1}{2}\left(\rho_{3}+\rho_{2}\right) \dot{u}_{3}^{2}+\rho_{w} \dot{u}_{3} \dot{w}_{3}+\frac{1}{2} m_{3} \dot{w}_{3}^{2}+\frac{1}{2} \rho_{1} \dot{u}_{2}^{2}
$$

with

$$
\left(m_{i j}\right)_{3}=\rho_{w} \iiint_{\Omega} \sum_{k} \alpha_{k i}^{(3)} \alpha_{k j}^{(3)} d \Omega=\left(m_{j i}\right)_{3}
$$

and

$$
\left(m_{i j}\right)_{3}=m_{3} \delta_{i j} .
$$

For the general case $\dot{u}_{1} \neq \dot{u}_{2} \neq \dot{u}_{3}$, the pore may be thought of as a mechanical system obtained from the combination of two fictitious systems: one without water-ice relative motion and another one without water-solid relative motion. If these two fictitious systems were independent, the kinetic energy $C$ of the global system would be given by the simple sum $\mathbf{C}_{1}+\mathbf{C}_{3}$. Nevertheless, they are coupled by water from the energy point of view. Hence the kinetic energy contribution of water must be considered only once in the global system and the expression of $\mathbf{C}$ is given by

$$
\mathrm{C}=\mathrm{C}_{1}+\mathrm{C}_{3}-\frac{1}{2}\left(\rho_{1}+\rho_{2}+\rho_{3}\right) \dot{u}_{2}^{2} .
$$

Expressed as a function of $\dot{u}_{1}, \dot{u}_{2}$ and $\dot{u}_{3}, \mathrm{C}$ is written as

$$
\begin{aligned}
\mathrm{C}= & \frac{1}{2}\left(\rho_{1}-\rho_{2}+m_{1} \phi_{w}^{2}\right) \dot{u}_{1}^{2}+\left(\rho_{2}-m_{1} \phi_{w}^{2}\right) \dot{u}_{1} \dot{u}_{2} \\
& +\frac{1}{2}\left(m_{1} \phi_{w}^{2}+m_{3} \phi_{w}^{2}-\rho_{2}\right) \dot{u}_{2}^{2}+\left(\rho_{2}-m_{3} \phi_{w}^{2}\right) \dot{u}_{2} \dot{u}_{3} \\
& +\frac{1}{2}\left(\rho_{3}-\rho_{2}+m_{3} \phi_{w}^{2}\right) \dot{u}_{3}^{2} .
\end{aligned}
$$

\section{B. Expressions of the generalized mass densities $\rho_{l j}$}

The generalized mass densities $\rho_{i j}$ terms are obtained from the identification of the coefficients of expression (8) to those of expression (28):

$$
\rho_{11}=\phi_{s} \rho_{s}+\left(a_{12}-1\right) \phi_{w} \rho_{w},
$$

$$
\begin{aligned}
& \rho_{12}=-\left(a_{12}-1\right) \phi_{w} \rho_{w}, \\
& \rho_{22}=\left(a_{12}+a_{23}-1\right) \phi_{w} \rho_{w}, \\
& \rho_{23}=-\left(a_{23}-1\right) \phi_{w} \rho_{w}, \\
& \rho_{33}=\phi_{i} \rho_{i}+\left(a_{23}-1\right) \phi_{w} \rho_{w},
\end{aligned}
$$

where

$$
a_{12}=\frac{m_{1} \phi_{w}}{\rho_{w}}=\phi_{w} \iiint_{\Omega} \sum_{k}\left(\alpha_{k k}^{(1)}\right)^{2} d \Omega=a_{21}
$$

and

$$
a_{23}=\frac{m_{3} \phi_{w}}{\rho_{w}}=\phi_{w} \iiint_{\Omega} \sum_{k}\left(\alpha_{k k}^{(3)}\right)^{2} d \Omega=a_{32} .
$$

It may be noticed that when there is no relative motion between the three phases one may define an average mass density as

$$
\rho=\rho_{11}+2 \rho_{12}+\rho_{22}+2 \rho_{23}+\rho_{33}=\phi_{s} \rho_{s}+\phi_{w} \rho_{w}+\phi_{i} \rho_{i}
$$

corresponding to the frozen porous medium effective mass density.

\section{Determination of coefficients $a_{12}$ and $a_{23}$}

Here again, we may consider the particular case where no relative motion exists between ice and water and the particular case where no relative motion exists between solid and water in order to determine the coefficients $a_{12}$ and $a_{23}$. Assuming no direct contact between solid and ice and no water-ice relative motion the solid matrix is surrounded by a fluidlike medium whose mass density is given by

$$
\rho=\left(\phi_{w} \rho_{w}+\phi_{i} \rho_{i}\right) /\left(\phi_{w}+\phi_{i}\right) .
$$

This problem is equivalent to the problem of inertial coupling in a fluid saturated porous medium and has been already studied (Biot, 1956a). The inertial drag parameter $a_{12}$ of the order of tortuosity is deduced using the results given by Berryman (1980):

$$
a_{12}=r_{12}\left(\phi_{s} \rho\right) /\left(\phi_{w} \rho_{w}\right)+1
$$

where $r_{12}$ characterizes the geometrical aspect of the boundary separating solid from water. Let us recall that $r_{12}=\frac{1}{2}$ for spheres (see Lamb, 1945 or Landau and Lifchitz, 1969). Similarly, $a_{23}$ is given as a function of the geometrical parameter $r_{23}$ characterizing the ice-water boundary by

$$
a_{23}=r_{23}\left(\phi_{i} \rho^{\prime \prime}\right) /\left(\phi_{w} \rho_{w}\right)+1
$$

with

$$
\rho^{\prime \prime}=\left(\phi_{w} \rho_{w}+\phi_{s} \rho_{s}\right) /\left(\phi_{w}+\phi_{s}\right) .
$$

\section{DETERMINATION OF THE FRICTION COEFFICIENTS $b_{11}$ AND $b_{33}$}

\section{A. Connection with Darcy's law}

The friction coefficients $b_{11}$ and $b_{33}$ may be determined using the expression (11) of the dissipation potential. If $b_{33}=0$, one finds the dissipation potential corresponding to a 
single phase flow of a fluid in a porous solid. Using Darcy's law (see Bourbié et al., 1986) $b_{11}$ is expressed as a function of the solid permeability $\kappa_{s}$ as

$$
b_{11}=\left(\eta_{w} \phi_{w}^{2} / \kappa_{s}\right)_{b_{33}=0} .
$$

If $b_{11}=0$, then defining an ice matrix permeability $\kappa_{i}, b_{33}$ is given by

$$
b_{33}=\left(\eta_{w} \phi_{w}^{2} / \kappa_{i}\right)_{b_{11}=0} .
$$

\section{B. Permeabilities of frozen porous media}

The permeability of frozen porous media is not a wellknown parameter. In this simple model, the two permeabilities defined are assumed to obey the Kozeny-Carman relationship (see Berryman, 1980). Considering the definitions (29) and (30), $\kappa_{i}$ must be infinite for $b_{33}$ to be zero: $\kappa_{s}$ $=\left(\kappa_{s}\right)_{\kappa_{i} \rightarrow \infty}$. This would correspond to a situation where water would flow along ice without friction. The solid permeability $\kappa_{s 0}$ for a water proportion $1-\phi_{s}=\varepsilon$ corresponding to a totally defrosted medium is supposed to be known. A partially frozen medium contains a proportion $\phi_{w}$ of unfrozen water. In this case an expression of the solid permeability is deduced using Kozeny-Carman's relationship

$$
\kappa_{s}=\kappa_{s 0} \phi_{w}^{3} / \varepsilon^{3} .
$$

Similarly, $\kappa_{i}$ is defined as the permeability of the ice matrix for a flow of water along solid without friction: $\kappa_{i}$ $=\left(\kappa_{i}\right)_{\kappa_{s} \rightarrow \infty}$. When the medium is totally frozen, the ice forms a matrix of apparent porosity $1-\varepsilon$. In this situation the ice permeability $\kappa_{i 0}$ is supposed to be known. The apparent porosity of the ice rnatrix for a partially frozen medium is $1-\phi_{i}$. In this case the ice permeability is obtained using Kozeny-Carman's relationship

$$
\kappa_{i 1} \phi_{i}^{2} /\left(1-\phi_{i}\right)^{3}=\kappa_{i 0} \varepsilon^{2} / \phi_{s}^{3} .
$$

In fact the apparent porosity is filled with water and solid. However, only the water proportion $\phi_{w}$ must be considered since only water may flow. Using a formula similar to the one used for the solid

$$
\kappa_{i}=\kappa_{i 1} \phi_{w}^{3} /\left(1-\phi_{i}\right)^{3},
$$

one may obtain an expression for the ice permeability as:

$$
\kappa_{i}=\kappa_{i 0}\left(\varepsilon / \phi_{i}\right)^{2}\left(\phi_{w} / \phi_{s}\right)^{3} .
$$

It can be noticed that as $\kappa_{s}$ and $\kappa_{i}$ characterizing the relative water-solid flows and water-ice flows may be designated as relative permeabilities. However this definition is not consistent with the usual relative permeabilities defined for the study of viscous flows of several mixed fluids in a porous medium (see Bourbié et al., 1987). $\kappa_{i}$ might be supposed to be higher than $\kappa_{s}$ because ice proportion which is generally less than 0.4 acts as $\left(1 / \phi_{i}\right)^{2}$ in Eq. (31). Considering Eq. (11) it is possible to define an effective permeability $\kappa_{\text {eff }}$ for frozen porous media in the particular case of $\dot{u}_{1}=\dot{u}_{3}=0$ :

$$
\mathbf{D}=\frac{1}{2}\left(b_{11}+b_{33}\right)\left|\dot{u}_{2}\right|^{2}=\frac{1}{2} \frac{\eta_{w}}{\kappa_{\text {eff }}}\left|\phi_{w} \dot{u}_{2}\right|^{2},
$$

where the effective permeability is given by

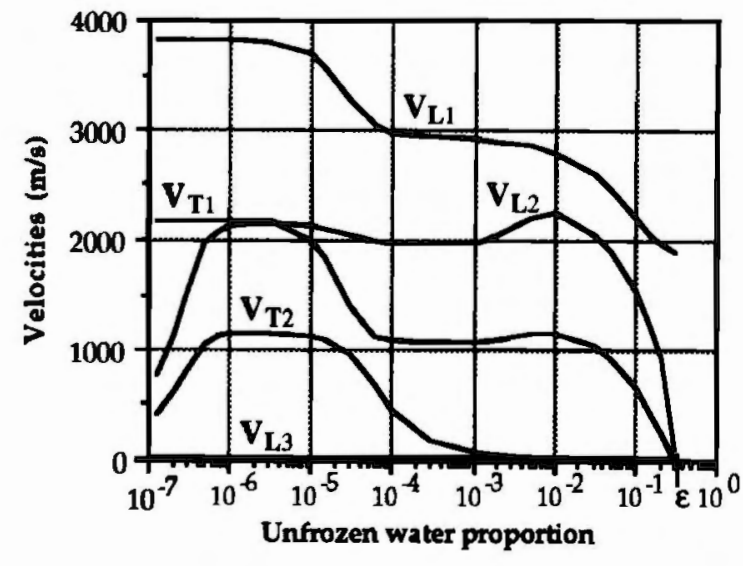

FIG. 3. Longitudinal and transverse wave velocities as functions of unfrozen water proportion for a nonconsolidated medium (glass powder). The indices 1, 2, 3 used here refer to solutions of Eqs. (20) and (21), and not to the material phases (solid, water, and ice).

$$
\kappa_{\mathrm{eff}}=\left(1 / \kappa_{\mathrm{s}}+1 / \kappa_{i}\right)^{-1} .
$$

For a constant water flow in the partially frozen medium, $\phi_{w} \dot{u}_{2}$ would be the filtration velocity and $\kappa_{\text {eff }}$ the permeability that would be measured experimentally. Assuming that $\kappa_{i}$ is large with respect to $\kappa_{s}$, the effective permeability would be given by:

$$
\kappa_{\mathrm{eff}}=\kappa_{s}=\kappa_{s 0}\left(\phi_{w} / \varepsilon\right)^{3} .
$$

This expression is similar to the one proposed by O'Neill and Miller (1985) with the difference that these authors have considered a variable exponent $n$ of the order of 8 . Here the exponent 3 is a consequence of the use of Kozeny-Carman's relationship in our simple model.

\section{NUMERICAL SOLUTION OF THE EQUATIONS OF PROPAGATION}

The characteristic equations (20) and (21) are solved numerically using Newton's method with complex coefficients to provide the wave velocities and attenuation coefficients of three longitudinal modes noted L1, L2, L3, and of the two transverse modes T1 and T2 as functions of unfrozen water volumic content. Figures 3 and 4 show the calculated

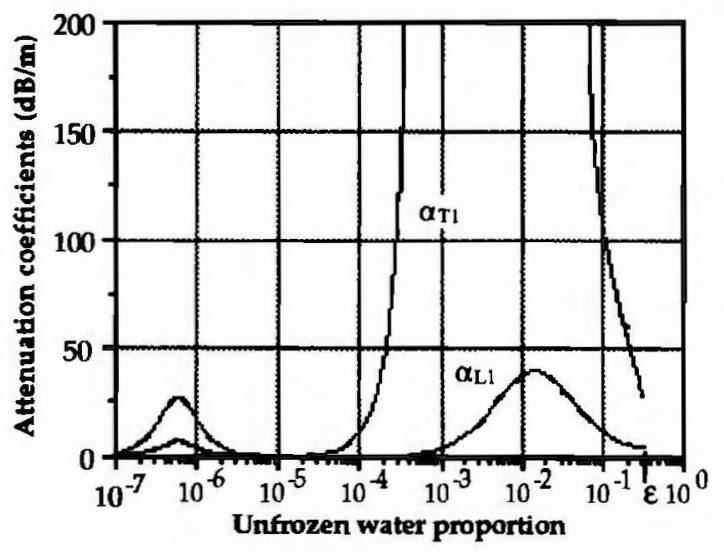

FIG. 4. Attenuation coefficients of $\mathrm{Ll}$ and $\mathrm{T} 1$ modes as functions of unfrozen water proportion (glass powder). 


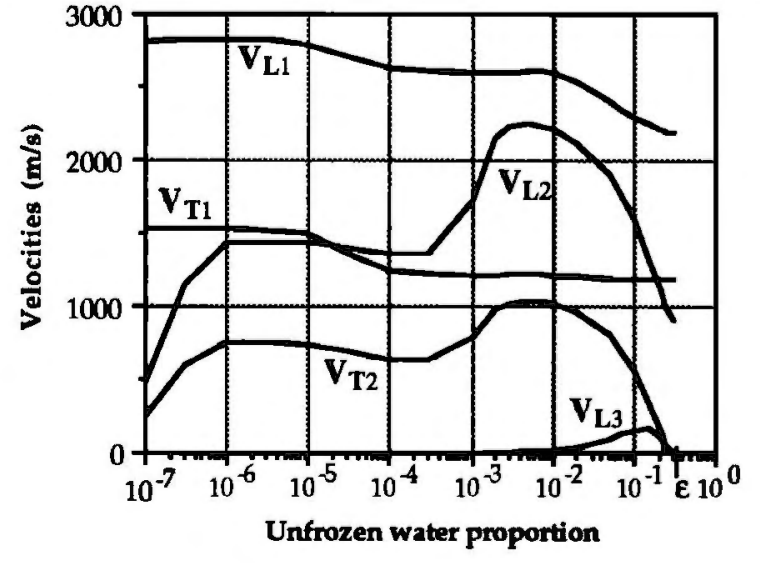

FIG. 5. Longitudinal and transverse wave velocities as functions of unfrozen water proportion for a consolidated medium (sintered bronze). The indices 1,2,3 used here refer to solutions of Eqs. (20) and (21), and not to the material phases (solid, water, and ice).

curves of velocities and attenuation coefficients at $500 \mathrm{kHz}$ in the example of a nonconsolidated medium made of fine glass powder saturated with water. The constants chosen for the calculation are given as follows:

Porosity: $\varepsilon=0.305$, average radius of solid grains: 60 $\mu \mathrm{m}$, frequency: $f=500 \mathrm{kHz}$,

$\rho_{w}=1000 \mathrm{~kg} / \mathrm{m}^{3}, K_{w}=2.25 \times 10^{9} \mathrm{~Pa}$, water viscosity: $\eta_{w}=1.8 \times 10^{-3} \mathrm{~kg} \mathrm{~m}^{-1} \mathrm{~s}^{-1}$ (at $0^{\circ} \mathrm{C}$ ),

$\rho_{i}=920 \mathrm{~kg} / \mathrm{m}^{3}, K_{i}=8.5 \times 10^{9} \mathrm{~Pa}, \mu_{i}=3.6 \times 10^{9}$ Pa (see Roethlisberger, 1972),

Initial permeability of ice matrix (for $\phi_{i}=\varepsilon=0.305$ ): $\kappa_{i 0}=5 \times 10^{-4} \mathrm{~m}^{2}$,

$\rho_{s}=2480 \mathrm{~kg} / \mathrm{m}^{3}, \quad K_{s}=5 \times 10^{10} \mathrm{~Pa}, \mu_{s}=3 \times 10^{10} \mathrm{~Pa}$,

$K_{\mathrm{sm}} \approx 0 \mathrm{~Pa}, \mu_{\mathrm{sm}} \approx 0 \mathrm{~Pa}$,

Solid permeability: $\kappa_{s 0} \approx 10^{-11} \mathrm{~m}^{2}$.

Figures 5 and 6 show the results for sintered bronze beads as a consolidated medium. The constants chosen for the calculation are given as follow:

Porosity: $\varepsilon=0.30$, average radius of solid grains: $70 \mu \mathrm{m}$, frequency: $f=500 \mathrm{kHz}$,

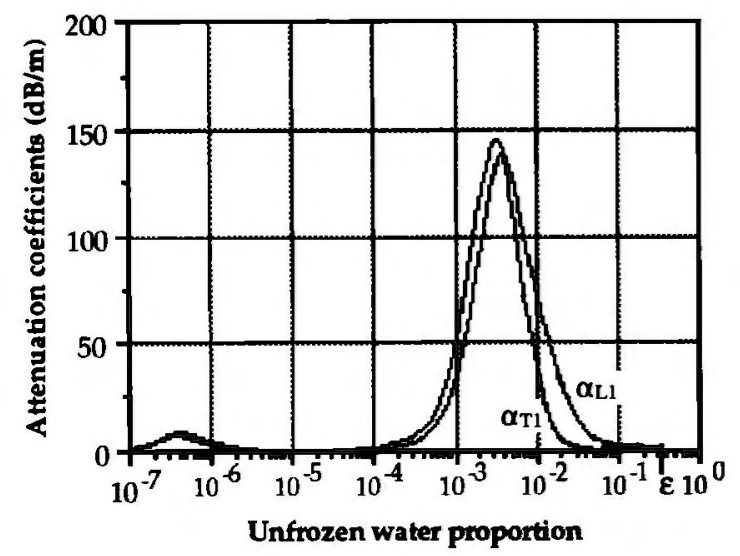

FIG. 6. Attenuation coefficients of $\mathrm{L} 1$ and $\mathrm{T} 1$ modes as functions of unfrozen water proportion (sintered bronze). $\rho_{w}=1000 \mathrm{~kg} / \mathrm{m}^{3}, K_{w}=2.25 \times 10^{9} \mathrm{~Pa}$, water viscosity: $\eta_{w}=1.8 \times 10^{-3} \mathrm{~kg} \mathrm{~m}^{-1} \mathrm{~s}^{-1}$ (at $0^{\circ} \mathrm{C}$ ),

$\rho_{i}=920 \mathrm{~kg} / \mathrm{m}^{3}, K_{i}=8.5 \times 10^{9} \mathrm{~Pa}, \mu_{i}=3.6 \times 10^{9} \mathrm{~Pa}$ (see

Roethlisberger, 1972),

Initial permeability of ice matrix (for $\phi_{i}=\varepsilon=0.30$ ): $\kappa_{i 0}=10^{-3} \mathrm{~m}^{2}$,

$\rho_{s}=8774 \mathrm{~kg} / \mathrm{m}^{3}, K_{s}=1.46 \times 10^{11} \mathrm{~Pa}, \mu_{\mathrm{s}}=4.01 \times 10^{10} \mathrm{~Pa}$, $K_{\text {sm }}=1.28 \times 10^{10} \mathrm{~Pa}, \mu_{\mathrm{ms}}=8.84 \times 10^{9} \mathrm{~Pa}$.

Solid permeability: $\kappa_{s 0} \approx 3.10^{-11} \mathrm{~m}^{2}$.

In the example of a nonconsolidated medium, the velocities (Fig. 3) of L2, L3, T1, and T2 modes tend to zero when the unfrozen water proportion tends to the porosity and one obtains the single longitudinal mode predicted by Biot. The calculated velocity of $\mathrm{L} 3$ mode is very low with respect to those of the other modes. For the consolidated medium, the velocities (Fig. 5) of the $\mathrm{L} 2$ and $\mathrm{T} 2$ tend, respectively, to the velocities of Biot's slow wave and of shear wave when the medium is totally defrosted. In the two cases, the peculiar shape of the velocity curves is a consequence of the modelization of elastic and inertial coupling. Indeed, as two eigenvalues of the characteristic equation giving the velocities shift and tend to take the same value, the coupling effect tends to separate them and thus prevents the crossing of the velocity curves.

Figures 4 and 6 show the attenuation curves as functions of unfrozen water proportion. The role of the parameters that govern viscous attenuation being not well known and not having a good model for the permeability of frozen media, only the results for $\mathrm{L} 1$ and $\mathrm{T} 1$ modes are presented in a qualitative way. The two peaks of attenuation appearing for each mode are explained qualitatively by the variations of viscous attenuation as a function of the variable permeabilities of our model. It is natural to think that viscous attenuation is low in a high permeability material since the fluid may flow without friction. On the other hand, fluid flow is impossible in a zero permeability material and attenuation of the viscous type must also be zero in this case. Between these two limit cases, attenuation may take a maximum value for a given value of permeability. The existence of two permeabilities in our model implies the presence of two attenuation peaks for each mode. It is interesting to notice that the principal maximum of attenuation occurs as the thickness of unfrozen water layer around the solid grains approaches of the viscous skin depth.

Figure 7 shows the Poisson's ratio calculated from the L1 and T1 modes. The range of variation for the consolidated medium appears relatively small. The Poisson's ratio of the unconsolidated medium tends to 0.5 as expected when the ice is no longer present to give a shear strength to the medium.

Figures 8 and 9 show the frequency variations of the L1 mode calculated for three unfrozen water proportions for the consolidated medium. It appears that the L1 mode velocity increases slightly with frequency (Fig. 8).

Observing the attenuation curve of Fig. 9, one may notice that the characteristic frequency separating the low frequency regime from the higher frequency regime shifts as the unfrozen water content changes. Given by the abscissa corresponding to a change of curvature on the attenuation 


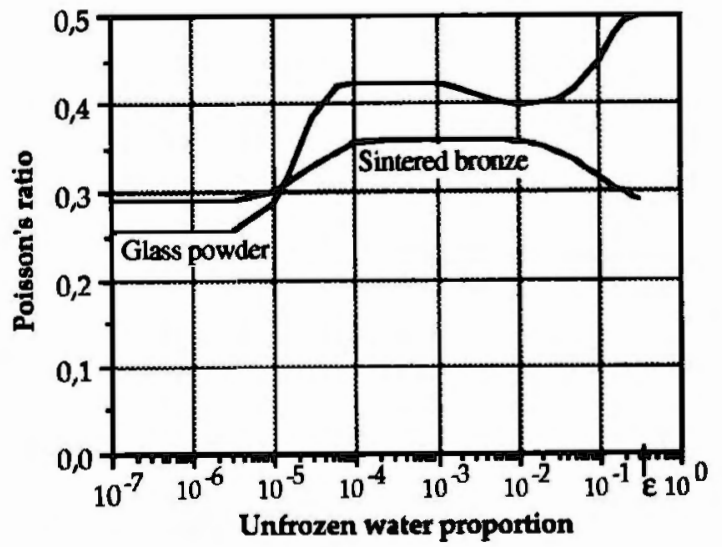

FIG. 7. Poisson's ratio calculated from the $\mathrm{L} 1$ and $\mathrm{T} 1$ mode velocities for a nonconsolidated medium (glass powder) and for a consolidated medium (sintered bronze).

curve, the characteristic frequency is of the order of $300 \mathrm{kHz}$ for $\phi_{w}=5 \times 10^{-3}$ and $75 \mathrm{kHz}$ for $\phi_{w}=10^{-2}$. This is a consequence of the variation of unfrozen water thickness as a function of unfrozen water content. In fact, Biot's characteristic frequency varies as the inverse of the square radius of the capillary pore. In our problem the pore diameter is replaced by the thickness $h$ of the water film separating solid from ice. Recalling that $h=r_{s}\left[\left(1+\phi_{w} / \phi_{s}\right)^{1 / 3}-1\right], h$ may be simplified as $h \approx\left(r_{s} \phi_{w}\right) /\left(3 \phi_{s}\right)$ if $\phi_{w} \ll \phi_{s}$. Since it varies as $h^{-2}$ the characteristic frequency must be approximately four times less for $\phi_{w}=10^{-2}$ than for $\phi_{w}=5 \times 10^{-3}$. For $\phi_{w}=10^{-3}$, this frequency is translated to a higher value of $1.2 \mathrm{MHz}$.

The calculation of the frequency variation of the $\mathrm{T} 1$ mode brings out similar results as for the L1 mode: a slight dispersion with a small increase with frequency and the two frequency regimes for the attenuation separated by a characteristic frequency depending on the unfrozen water film thickness.

With the help of 'Thomson's formula (see Aguirre-Puente and Bernard, 1978) and porosimetric distribution, it is possible to determine a thermodynamical relationship between unfrozen water proportion and temperature. Thomson's for-

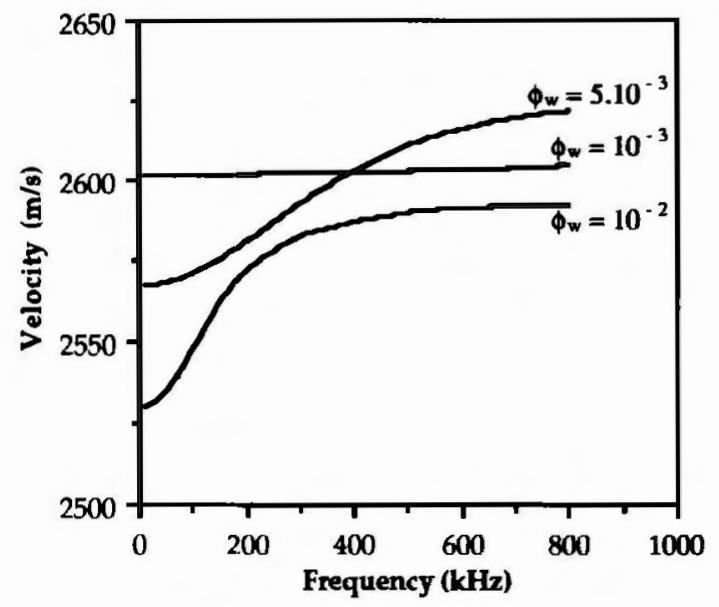

FIG. 8. Frequency variations of the $\mathrm{Ll}$ mode velocity for three values of unfrozen water proportion (sintered bronze).

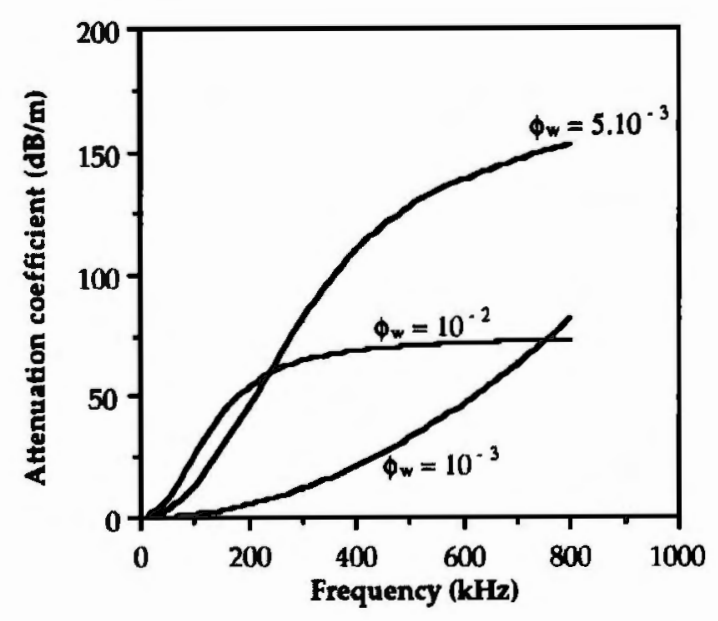

FIG. 9. Attenuation coefficient of the L1 mode as a function of frequency for three values of unfrozen water proportion (sintered bronze).

mula gives the equilibrium temperature $T$ at the ice-water interface in a capillary pore with radius $r$

$$
\ln \frac{T}{T_{0}}=-\frac{2 \sigma_{i w}}{r} \frac{1}{\rho_{i} L_{w}},
$$

where $\sigma_{i w}=3.5 \times 10^{-2} \mathrm{~N} \mathrm{~m}^{-1}$ is the surface tension at the interface, $\rho_{i}=920 \mathrm{~kg} \mathrm{~m}^{-3}, L_{w}=3.337 \times 10^{5} \mathrm{~J} \mathrm{~kg}^{-1}$ is the latent ice fusion heat and $T_{0}=0{ }^{\circ} \mathrm{C}=273 \mathrm{~K}$. The unfrozen water proportion $\phi_{w}$ may be written as the product of the porosity $\varepsilon$ and of the distribution function $F(r)$ giving the pore fraction of which radii are greater than $r$. Thus

$$
\phi_{w}=\varepsilon F(r)=\varepsilon \int_{-\infty}^{r_{0} / \ln \left(T_{0} / T\right)} f(r) d r, \text { with } r_{0}=\frac{2 \sigma_{i w}}{\rho_{i} L_{w}},
$$

with $f(r)$ the probability density function being the derivative of $F(r)$. Assuming a Gaussian porosimetric distribution, we evaluate in Fig. 10, the unfrozen water proportion for an average radius $r_{\mathrm{av}}=0.1 \mu \mathrm{m}$, for several standard deviations $\Delta r$, and for a porosity $\varepsilon=0.3$. For a given medium, one may then obtain velocities as function of temperature. Figure 11 shows the velocities of $\mathrm{L} 1, \mathrm{~L} 2, \mathrm{~T} 1$, and $\mathrm{T} 2$ modes calculated at $500 \mathrm{kHz}$ as functions of temperature for the example of the nonconsolidated medium. The parameters chosen for the

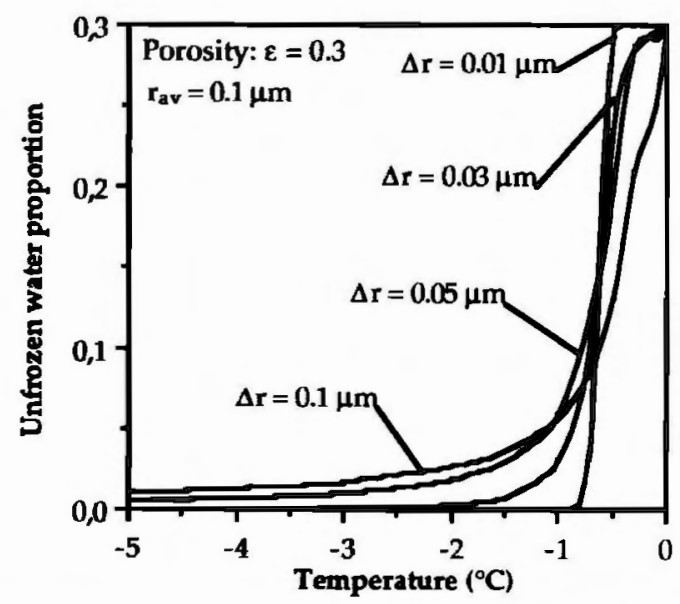

FIG. 10. Unfrozen water proportion calculated with Thomson's formula for a Gaussian porosimetric distribution. 


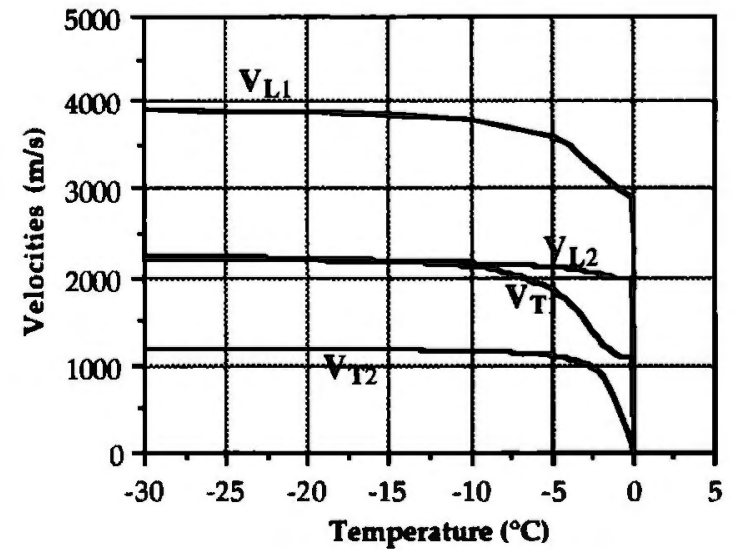

FlG. 11. Velocities of the $\mathrm{L} 1, \mathrm{~L} 2, \mathrm{~T} 1$, and $\mathrm{T} 2$ modes calculated as functions of temperature for the nonconsolidated medium (glass powder).

calculation $\left(r_{\mathrm{av}}=10 \mu \mathrm{m}, \Delta r=4 \mu \mathrm{m}\right)$ are such that a great variation of water proportion is predicted between 0 and $-1{ }^{\circ} \mathrm{C}$. This may explain the peculiar discontinuous aspect of the velocity curves between these temperatures that seem anomalous.

\section{EXPERIMENTS}

Experiments were performed in the laboratory using one consolidated medium: a block of sintered bronze beads whose granulometry is of the order of $160 \mu \mathrm{m}$ and two nonconsolidated media: a glass powder and Caen silt whose granulometry are, respectively, of the order of 100 and 30 $\mu \mathrm{m}$. The water-saturated sample is quickly taken from ambient to $-30^{\circ} \mathrm{C}$. During a $10 \mathrm{~h}$ slow reheating, temperature is monitored by thermocouples located, in or upon, the sample and acoustical measurements are performed consisting in the transmission of short acoustical pulses through the sample. The transducers' nominal frequency is $500 \mathrm{kHz}$ and the ultrasonic path length in the sample is around $2 \mathrm{~cm}$. For the consolidated medium, the smaller size of the samples required the use of smaller transducers with nominal frequency of $5 \mathrm{MHz}$. The signals are displayed on a sampling

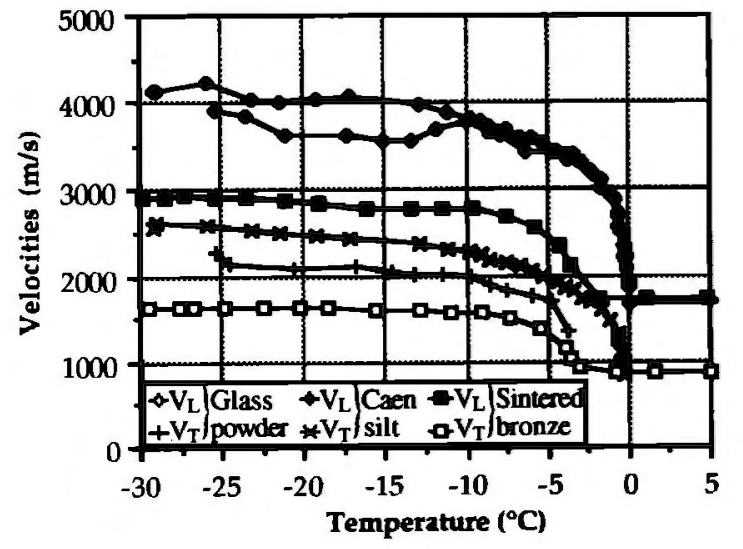

FIG. 12. Experimental velocities of the fastest modes in different materials as functions of temperature.

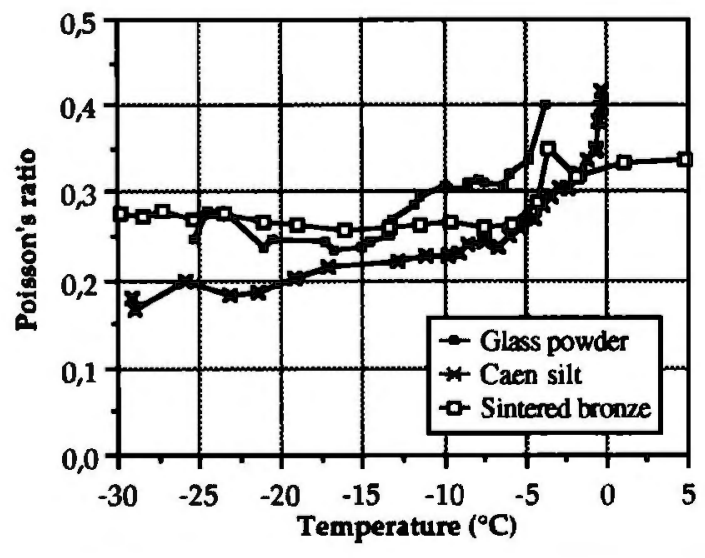

FIG. 13. Poisson's ratio as a function of temperature for different materials.

oscilloscope, digitized with an 8-bit $\mathrm{A} / \mathrm{D}$ converter and recorded on a microcomputer which allows the signal analysis in time or in frequency domain.

\section{A. Experimental velocities and Poisson's ratio}

Experimental longitudinal and transverse velocities of the fastest modes are plotted as functions of temperature in Fig. 12. The measured velocities at low temperature are comparable to those calculated for complete freezing. In the case of glass powder and Caen silt for complete defreezing, the wave attenuation caused by a small volume of air in the sample made the velocity measurements impossible. For sintered bronze, the measured velocities are smaller than those in glass powder or in Caen silt samples as expected since bronze has a high mass density. Nevertheless, at ambient temperature the measured velocity is less than the predicted one. This may be another consequence of the presence of air in the sample. Poisson's ratios calculated from the experimental velocities are shown in Fig. 13. The variation for sintered bronze is rather small, as expected. For glass powder and Caen silt samples the Poisson's ratio tends to 0.5 as expected when temperature tends to $0^{\circ} \mathrm{C}$.

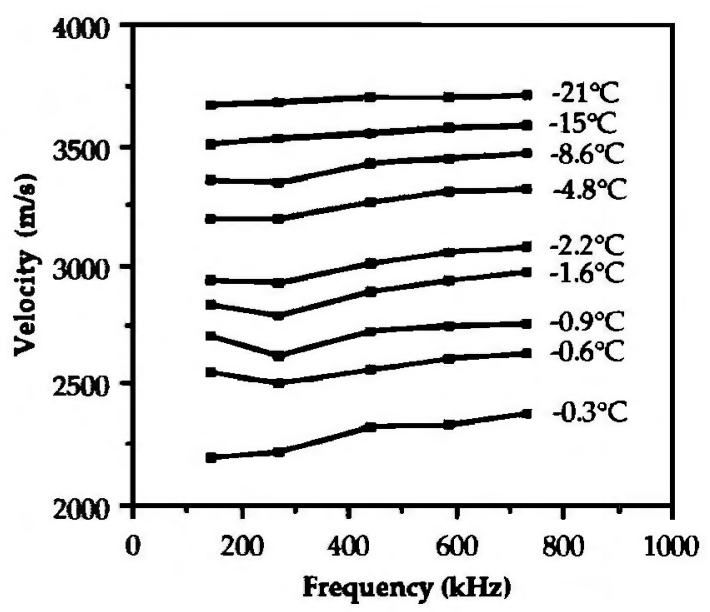

FIG. 14. Dispersion of the fastest longitudinal mode at different temperatures (in glass powder). 


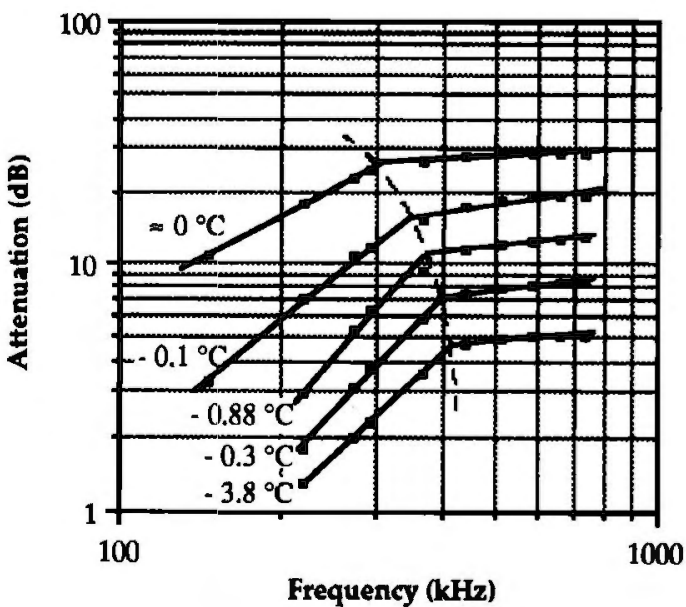

FIG. 15. Log-log plot of the frequency variations of the longitudinal wave attenuation as functions of temperature.

\section{B. Frequency variation of velocities}

The received signals were analyzed to determine the frequency variations of the velocities. Figure 14 shows the dispersion of the longitudinal mode at different temperatures for the glass powder sample. One can observe a slight dispersion with a global increase of velocity with frequency as expected. Similar results were obtained for the transverse wave.

\section{Attenuation measurements}

Attenuation is an increasing function of frequency as may be seen in Fig. 15 for longitudinal waves in the case of glass powder. The amplitudes have been normalized to the value obtained at the lowest available temperature of $-30^{\circ} \mathrm{C}$. The two frequency regimes given by Biot's theory may be observed in this log-log plot. The average slope is 2.1 ( 2 in Biot's theory) at low frequencies and 0.34 ( 0.5 in Biot's theory) in the higher frequency regime. As expected, the characteristic frequency shifts to lower frequencies as temperature increases (see the dotted curve).

\section{CONCLUSION}

The extension of Biot's theory to frozen media presented here shows the possibility of treating in a unified way the propagation in the different consolidated or nonconsolidated media met in practical situations. The formulation was made possible by making the physical assumption of no direct contact between the solid substrate and ice. Three longitudinal and two transverse modes are predicted; their velocities and attenuations are calculated numerically as functions of the unfrozen water proportion. The calculation of mechanical properties as functions of the thermodynamical state of the porous medium is possible with the help of a simple thermodynamical argument developed independently from the acoustical model. Experiments are performed in consolidated and unconsolidated samples. For the velocities, the experimental results are in good agreement with the theoretical ones. For the attenuations, some improvements to the model appear necessary. However, other experiments are necessary in order to confirm the theoretical predictions. In particular, investigations are in progress on the existence of possible secondary modes.

Aguirre-Puente, J., and Bernard, J. J. (1978). "Comportement au gel des matériaux de construction," (Le comportement thermique des matériaux de construction), Editions du Batiment et des Travaux Publics, pp. 29-72.

Arnold, V. (1974). Méthodes Mathématiques de la Mécanique Classique (Mir, Moscow).

Bacri, J. C., and Salin, D. (1982). "Propagation du son dans les milieux poreux saturés," Rev. Cethedec 74, 11-20.

Berryman, J. G. (1979). "Theory of elastic properties of composite materials," Appl. Phys. Lett. 35, 856-858.

Berryman, J. G. (1980). "Confirmation of Biot's theory," Appl. Phys. Lett. 37, 382-384

Biot, M. A. (1956a). "Theory of propagation of elastic waves in a fluid saturated porous solid. P. L. Low frequency range," J. Acoust. Soc. Am. 28, $168-178$.

Biot, M. A. (1956b). "Theory of propagation of elastic waves in a fluid saturated potous solid. P. 11. Highet frequency range," J. Acoust. Soc. Am. 28, 179-191.

Biot, M. A., and Willis, D. G. (1957). "The elastic coefficients of the theory of consolidation," J. Appl. Mech. 24, 594-601.

Biot, M. A. (1962). "Mechanics of deformation and acoustic propagation in porous media," J. Appl. Phys. 33, 1482-1498.

Bourbié, T., Coussy, O., and Zinszner, B. (1987). Acoustics of Porous Media (Technip, Paris).

Budiansky, B. (1965). "On the elastic moduli of some heterogeneous materials," J. Mech. Phys. Solids 13, 223-227.

Deschatres, M. H., Cohen-Ténoudji, F., Aguirre-Puente, J., and Thimus, J. F. (1989). "Ultrasonic propagation through frozen porous media. Liquid phase content determination," Ultrasonic Int. Conf. Proc. 158-163.

De Gennes, P. G. (1976). "On a relation between percolation theory and the elasticity of gels," J. Phys. Lett. 37, L1-L2.

Defay, R., and Prigogine, I. (1957). Surface Tension and Adsorption (Everett, Longmans-Green, London).

Deptuck, D., Harrison, J. P., and Zawadzki, P. (1985). "Measurement of elasticity and conductivity of a three-dimensional percolation system," Phys. Rev. Lett. 54(9), 913-916.

Gilpin, R. R. (1980). "Wire regelation at low temperatures," J. Colloïd Interface Sci. 77(2), 435-448.

Goldstein, H. (1980). Classical Mechanics (Addison-Wesley, Reading, MA).

Hashin, Z., and Shtrikman, S. (1963). "A variational approach to the theory of the elastic behaviors of multiphase material," J. Mech. Phys. Solids 11, 127-140.

Handbook of Chemistry and Physics (1965-1966). (Chemical Rubber Co., Boca Raton, FL), 46th ed.

Hill, R. (1965). "A self consistent mechanics of composite materials," J. Mech. Phys. Solids 13, 213-222.

Johnson, D. L., and Plona, T. J. (1982). "Acoustic slow waves and the consolidation transition," J. Acoust. Soc. Am. 72, 556-565.

Kittel, C. (1971). Introduction to Solid State Physics (Wiley, New York).

Kuster, G. T., and Toksöz, M. N. (1974). "Velocity and attenuation of seismic waves in two phase media. Part I, Theoretical formulation," Geophys. 39(5), 587-606.

Lamb, H. (1945). Hydrodynamics (Dover, New York).

Landau, L., and Lifchitz, E. (1969). Fluid Mechanics (Pergamon, London).

Leclaire, Ph. (1992). "Propagation acoustique dans les milieux poreux soumis au gel-Modélisation et expérience," Thèse de Doctorat en Physique, Université Paris 7, Paris.

Leclaire, Ph., Cohen-Ténoudji, F., and Aguirre-Puente, J. (1991). "Etude de la propagation acoustique dans les milieux poreux soumis au gelModélisation et expérience," XVIIIème Congrès Int. du Froid, Montréal, Canada.

Leclaire, Ph., Cohen-Ténoudji, F. and Aguirre-Puente, J. (1993). "A model of elastic wave propagation in frozen media. Comparison with experiments," Proc. Vlth Int. Conf. on Permafrost, Beijing, China.

O'Neill, K., and Miller, R. D. (1985). "Exploration of a rigid ice model of 
frost heave," Water Res. Res. 21(3), 281-296.

Peschel, G. (1968). "The viscosity of thin water films between two quartz plates," The International Union of Research and Testing of Materials and Structures (Chapman and Hall, London).

Plona, T. J., and Johnson, D. L. (1980). "Experimental study of the two compressional modes in water-saturated porous structures," Proc. IEEE Ultrason. Symp. 868-871

Roethlisberger, H. (1972). "Seismic exploration in cold region," CRREL Monograph II, A2A.

Timur, A. (1968). "Velocity of compressional waves in porous media at permafrost temperatures," Geophys. 33(4), 584-595. 